NOTICE: this is the author's version of a work that was accepted for publication in Systems and Control Letters. Changes resulting from the publishing process, such as peer review, editing, corrections, structural formatting, and other quality control mechanisms may not be reflected in this document. Changes may have been made to this work since it was submitted for publication. A definitive version was subsequently published in Systems and Control Letters, Vol. 61, no.1 (2012). DOI: 10.1016/j.sysconle.2011.10.018 


\title{
Coordination Control of Multiple Ellipsoidal Agents with Collision Avoidance and Limited Sensing Ranges
}

\author{
K.D. Do
}

\begin{abstract}
This paper contributes a design of cooperative controllers that force $N$ mobile agents with an ellipsoidal shape and a limited sensing range to track desired trajectories and to avoid collision between them. A separation condition for ellipsoidal agents is first derived. Smooth step functions are then introduced. These functions and the separation condition between the ellipsoidal agents are embedded in novel pairwise collision avoidance functions to design coordination controllers. The proposed control design guarantees: 1) smooth coordination controllers despite the agents' limited sensing ranges, 2) no collision between any agents, 3 ) asymptotical stability of desired equilibrium set, and 4) instability of all other undesired critical sets of the closed loop system.
\end{abstract}

Index Terms-Coordination control, ellipsoidal agents, collision avoidance, potential functions.

\section{INTRODUCTION}

Coordination control of multiple agents finds various applications to search, rescue, coverage, surveillance, reconnaissance and cooperative transportation. Therefore, a number of approaches has been available for coordination control of networked agents. Here, three popular methods are briefly mentioned. The leader-follower method (e.g., [1], [2], [3], [4]) uses several agents as leaders and others as followers. This method is easy to understand and ensures coordination maintenance if the leaders are disturbed. However, the desired coordination shape cannot be maintained if followers are perturbed unless a feedback is implemented, [5]. The behavioral method (e.g., [6], [7]), where each agent locally reacts to actions of its neighbors, is suitable for decentralized control but is difficult in control design and stability analysis since group behavior cannot explicitly be defined. The virtual structure method (e.g., [8], [9], [10]) treats all agents as a single entity. This method is amenable to mathematical analysis but is difficult to deal with a time-varying structure.

Research works on coordination control usually utilize one or more of the above methods in a centralized or a decentralized manner. Centralized strategies (e.g., [5], [11]) use a single controller that generates collision free trajectories in the workspace. These strategies guarantee a complete solution but require high computational power and are not robust. Decentralized schemes (e.g., [10], [12], [13], [14], [15], [16], [17], [18]) require less computational effort but have difficulties in controlling critical points, especially when collision avoidance

School of Mechanical Engineering, The University of Western Australia, 35 Stirling Highway, Crawley, WA 6009, Australia

Email: duc@mech.uwa.edu.au between the agents is a must. In all the above cited references, the shape of all the agents is considered as a single point or a circular disk or a sphere.

In practice, many agents such as submarines and rockets have a non-spherical, especially long and narrow, shape. If these agents are fitted to spheres, there is a problem with the large conservative volume. To illustrate this problem, we look at an example of fitting a cylindrical agent with a radius of $r_{c}$ and a length of $2 l_{c}$ to an ellipsoid with semiaxes of $a, b$ and $c$, and a sphere with a radius of $r_{s}$ as shown in Fig.1. By shrinking the space along the direction of the major axis of the ellipsoid, we can find $a=\sqrt{2} l_{c}$, $b=c=\sqrt{2} r_{c}$, and $r_{s}=\sqrt{r_{c}^{2}+l_{c}^{2}}$. Therefore, the conservative volume, $V_{c o n}$, defined as the difference between the volumes enclosed by the sphere and the ellipsoid, is given by $V_{\text {con }}=\frac{4 \pi r_{c}^{3}}{3}\left[\left(\frac{l_{c}^{2}}{r_{c}^{2}}+1\right) \sqrt{\frac{l_{c}^{2}}{r_{c}^{2}}+1}-2 \sqrt{2} \frac{l_{c}}{r_{c}}\right]$. This means that the conservative volume is always nonnegative and is proportional to cubic of the half length $l_{c}$ over the radius $r_{c}$ of an agent.

A spherical approximation of the shape of long and narrow agents can adversely affect performance of a coordination control algorithm. An example is the case where it is a must to force a group of long and narrow agents through a long and narrow passageway. In some cases, a spherical approximation of the agents' shape can result in failure of a coordina-

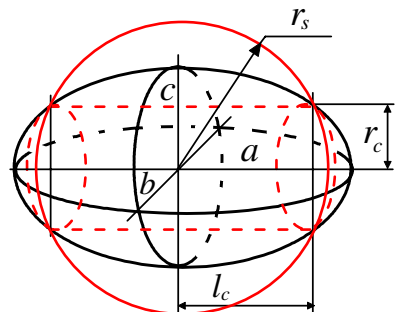

Fig. 1. Fitting a cylindrical agent to an ellipsoid and a sphere. tion control algorithm. As an illustration, we consider two cylindrical agents with lengths of $2 l_{1}$ and $2 l_{2}$, and radii of $r_{1}$ and $r_{2}$, respectively. Assuming that $r_{1}$ and $r_{2}$ are much less than $l_{1}$ and $l_{2}$, respectively, i.e., the two agents have a long and narrow shape. We now require these two agents to move cooperatively in a way that they do not collide with each other and the distance $d_{12}$ between them is such that $\left(r_{1}+r_{2}\right)+\epsilon_{12}<d_{12}<\left(l_{1}+l_{2}\right)-\epsilon_{12}$ with $\epsilon_{12}$ being a feasible positive constant. Clearly, a spherical approximation of the agents' shape is not applicable in this case for a coordination control algorithm. On the other hand, an ellipsoidal approximation can be applicable. In addition, an ellipsoidal approximation of the agents' shape for collision avoidance between the agents in a coordination control algorithm covers a spherical approximation of the agents' shape by setting the 
semi-axes of the ellipsoid equal, but not vice versa. The above discussion indicates that it is much more efficient to use an ellipsoidal approximation of the agents with a long and narrow shape for collision avoidance in designing coordination control algorithms.

Despite of the above advantages of an ellipsoidal approximation of the agents' shape, coordination control for ellipsoidal agents has not been addressed in the literature. This is partially due to difficulties in determining a separation condition between two ellipsoids. There have been two main methods to determine a separation condition between ellipsoids. The first method found in [19], [20] consists of determining the intersection of the ellipsoids with the plane containing the line joining their centers and rotating the plane. The distance of the closest approach [21] of the two ellipses formed by the intersection is a periodic function of the plane orientation, of which the maximum value corresponds to the closest distance between the two ellipsoids. The second method [22] found the condition for separation between two ellipsoids is based on the discriminant of there characteristic polynomial. Both methods are too complicated for an application in coordination control. If these methods are applied for collision avoidance, the condition, for which the minimum distance between two disks or the discriminant of their characteristic polynomial is positive, is extremely complicated to be embedded in a proper potential function for designing a coordination control algorithm.

The aforementioned observations motivate contributions of this paper on a design of coordination controllers for ellipsoidal agents with limited sensing ranges. The objective is to design controllers to force the agents to track desired trajectories and to guarantee no collision between them. It is noted that the objective of the present work is different from those on formation control of multiple agents in [23], [24], [25], [26], [27]. In these papers, a set of agents, a graph topology specifying what agents sense each other and what parameter they sense, and a graph topology specifying what parameter (e.g., distance) agents want to control with respect to the what other agents are first given. Using this information only, a distributed controller is then designed for each agent to achieve its own constraints. The formation objective is to force the entire group to achieve a desired shape.

Our proposed design provides smooth coordination controllers despite the agents' limited sensing ranges, no collision between any agents, asymptotic stability of desired equilibrium set, and instability of all other undesired critical sets of the closed loop system. The paper's contributions include: 1) a new condition for separation between two ellipsoids, see Section II-A; 2) new smooth step functions; 3) new pairwise collision avoidance functions for two ellipsoidal agents, see Section II-A; and 4) a derivation of coordination controllers based on the pairwise potential functions, see Section IV-C.

\section{Preliminaries}

\section{A. Separation condition between two ellipsoids}

This section presents a condition for separation of two ellipsoids applicable for collision avoidance in the coordination

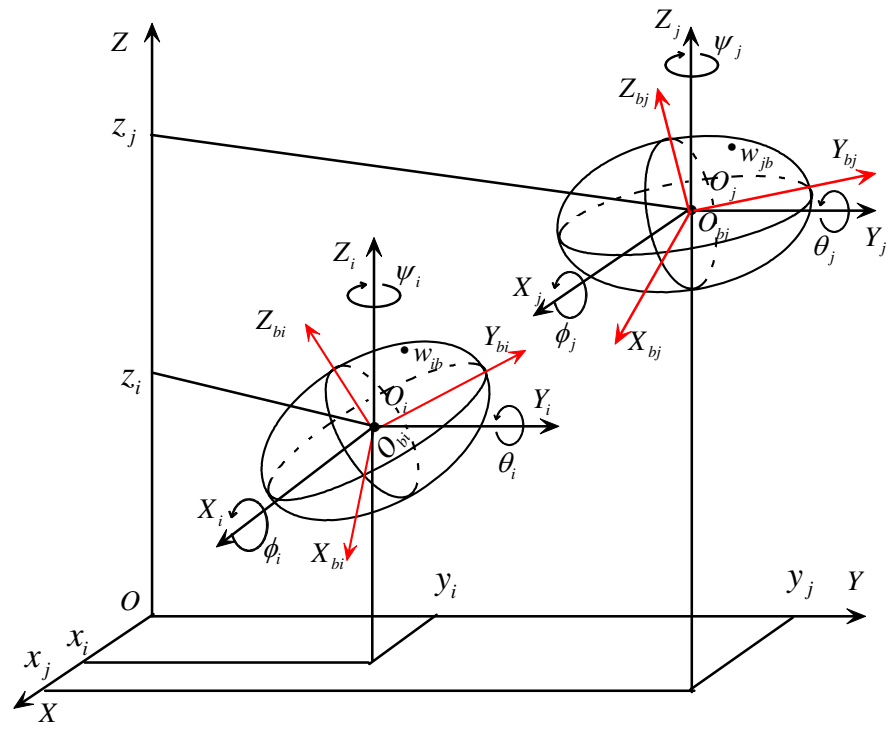

Fig. 2. Two ellipsoids and their coordinates.

control design later. As such, we consider two ellipsoids $i$ and $j$ shown in Fig. 2. In this figure, $O X Y Z$ is the earthfixed frame, $O_{i} X_{i} Y_{i} Z_{i}$ is the body-fixed frame attached to ellipsoid $i, \boldsymbol{q}_{i}=\left[x_{i} y_{i} z_{i}\right]^{T}$ denotes the position of the center $O_{i}$, and $\boldsymbol{\eta}_{i}=\left[\phi_{i} \theta_{i} \psi_{i}\right]^{T}$ denotes the orientation (roll, pitch and yaw angles) of the ellipsoid $i$. Moreover, $\left(a_{i}, b_{i}, c_{i}\right)$ denote the semi-axes of the ellipsoid $i$. These notations are similar for the ellipsoid $j$.

Lemma 2.1: Consider two ellipsoids $i$ and $j$, which have semi-axes of $\left(a_{i}, b_{i}, c_{i}\right)$ and $\left(a_{j}, b_{j}, c_{j}\right)$, and orientation vectors $\boldsymbol{\eta}_{i}=\left[\phi_{i} \theta_{i} \psi_{i}\right]^{T}$ and $\boldsymbol{\eta}_{j}=\left[\phi_{j} \theta_{j} \psi_{j}\right]^{T}$, and are centered at $\boldsymbol{q}_{i}=\left[x_{i} y_{i} z_{i}\right]^{T}$ and $\boldsymbol{q}_{j}=\left[x_{j} y_{j} z_{j}\right]^{T}$, respectively, see Fig. 2. Define the transformed distance $\Delta_{i j}$ between the ellipsoids $i$ and $j$ as

$$
\Delta_{i j}=\left\|\boldsymbol{Q}_{i j} \overline{\boldsymbol{q}}_{i j}\right\|-1
$$

where

$$
\begin{aligned}
& \boldsymbol{Q}_{i j}=\boldsymbol{I}_{3 \times 3}-\left(\boldsymbol{I}_{3 \times 3}+\kappa_{i j} \boldsymbol{T}_{j}\right)^{-1}, \\
& \overline{\boldsymbol{q}}_{i j}=\boldsymbol{P}_{i} \boldsymbol{q}_{i j},
\end{aligned}
$$

with $\boldsymbol{I}_{3 \times 3}$ being a $3 \times 3$ identity matrix. The vector $\boldsymbol{q}_{i j}$ denotes the relative position vector between the ellipsoids $i$ and $j$. The matrix $\boldsymbol{P}_{i}$ represents the so-called negative inverse of the rotational matrix of the ellipsoid $i$ by the angular vector $\boldsymbol{\eta}_{i}$ around its axes. The vector $\boldsymbol{q}_{i j}$ and the matrix $\boldsymbol{P}_{i}$ are given by

$$
\begin{aligned}
& \boldsymbol{q}_{i j}=\boldsymbol{q}_{i}-\boldsymbol{q}_{j}, \\
& \boldsymbol{P}_{i}=-\boldsymbol{A}_{i}^{-1} \boldsymbol{R}^{-1}\left(\boldsymbol{\eta}_{i}\right), \\
& \boldsymbol{A}_{i}=\operatorname{diag}\left(a_{i}, b_{i}, c_{i}\right) .
\end{aligned}
$$

The matrix $\boldsymbol{R}(\bullet)$ represents the three dimensional rotational matrix with respect to the vector $\bullet$. The matrix $\boldsymbol{T}_{j}$ is the system matrix of the ellipsoid $j$ when the ellipsoids $i$ and $j$ are transformed to the spherical-ellipsoidal coordinates detailed in 
Appendix A. It is given by

$$
\boldsymbol{T}_{j}=\left[\begin{array}{ccc}
T_{j 11} & T_{j 12} & T_{j 13} \\
T_{j 12} & T_{j 22} & T_{j 23} \\
T_{j 13} & T_{j 23} & T_{j 33}
\end{array}\right],
$$

where

$$
\begin{aligned}
& T_{j 11}=\varepsilon_{11}^{2}+\varepsilon_{21}^{2}+\varepsilon_{31}^{2}, T_{j 12}=\varepsilon_{11} \varepsilon_{12}+\varepsilon_{21} \varepsilon_{22}+\varepsilon_{31} \varepsilon_{32}, \\
& T_{j 22}=\varepsilon_{12}^{2}+\varepsilon_{22}^{2}+\varepsilon_{32}^{2}, T_{j 13}=\varepsilon_{11} \varepsilon_{13}+\varepsilon_{21} \varepsilon_{23}+\varepsilon_{31} \varepsilon_{33}, \\
& T_{j 33}=\varepsilon_{13}^{2}+\varepsilon_{23}^{2}+\varepsilon_{33}^{2}, T_{j 23}=\varepsilon_{12} \varepsilon_{13}+\varepsilon_{22} \varepsilon_{23}+\varepsilon_{32} \varepsilon_{33},
\end{aligned}
$$

with $\varepsilon_{m n}$ for $m=1,2,3$ and $n=1,2,3$ being the element $(m, n)$ of the matrix $\left(\boldsymbol{A}_{i}^{-1} \boldsymbol{R}\left(\boldsymbol{\eta}_{i j}\right) \boldsymbol{A}_{j}\right)^{-1}$ with

$$
\begin{aligned}
& \boldsymbol{\eta}_{i j}=\boldsymbol{\eta}_{i}-\boldsymbol{\eta}_{j}, \\
& \boldsymbol{A}_{j}=\operatorname{diag}\left(a_{j}, b_{j}, c_{j}\right) .
\end{aligned}
$$

The variable $\kappa_{i j}$ is the largest root (the right most root) of the shortest distance equation

$$
\overline{\boldsymbol{q}}_{i j}^{T}\left(\boldsymbol{I}_{3 \times 3}+\kappa_{i j} \boldsymbol{T}_{j}\right)^{-T} \boldsymbol{T}_{j}\left(\boldsymbol{I}_{3 \times 3}+\kappa_{i j} \boldsymbol{T}_{j}\right)^{-1} \overline{\boldsymbol{q}}_{i j}-1=0,
$$

where $\left(\boldsymbol{I}_{3 \times 3}+\kappa_{i j} \boldsymbol{T}_{j}\right)^{-T}$ denotes the transpose of $\left(\boldsymbol{I}_{3 \times 3}+\right.$ $\left.\kappa_{i j} \boldsymbol{T}_{j}\right)^{-1}$.

The two ellipsoids are externally separated, i.e., the ellipsoids are outside of each other and do not contact with each other like Fig. 2, if

$$
\Delta_{i j}>0
$$

Remark 2.1: The transformed distance $\Delta_{i j}$ is a smooth function of $\boldsymbol{q}_{i j}, \boldsymbol{\eta}_{i}$, and $\boldsymbol{\eta}_{i j}$. Alternatively, $\Delta_{i j}$ is a smooth function of $\overline{\boldsymbol{q}}_{i j}$ and $\boldsymbol{\eta}_{i j}$.

Proof. See Appendix A.

\section{B. Smooth step function}

This section gives a definition of the smooth step function followed by a construction of this function. The smooth step function is to be embedded in a pairwise potential function to avoid discontinuities in the control law due to the agents' limited sensing ranges in solving the collision avoidance problem.

Definition 2.1: A scalar function $h(x, \alpha, \beta, \gamma)$ is said to be a smooth step function if it possesses the following properties
1) $h(x, \alpha, \beta, \gamma)=0, \forall x \in(-\infty, \alpha]$,
2) $h(x, \alpha, \beta, \gamma)=1, \forall x \in[\beta, \infty)$,
3) $0<h(x, \alpha, \beta, \gamma)<1, \forall x \in(\alpha, \beta)$,
4) $h(x, \alpha, \beta, \gamma)$ is smooth,
5) $h^{\prime}(x, \alpha, \beta, \gamma)>0, \forall x \in(\alpha, \beta)$,
6) $h^{\prime \prime}(x, \alpha, \beta, \gamma)=0$ at $x=x^{*} \in(\alpha, \beta)$,

where $x \in \mathbb{R}, h^{\prime}(x, \alpha, \beta, \gamma)=\frac{\partial h(x, \alpha, \beta, \gamma)}{\partial x}, h^{\prime \prime}(x, \alpha, \beta, \gamma)=$ $\frac{\partial^{2} h(x, \alpha, \beta, \gamma)}{\partial x^{2}}, \alpha$ and $\beta$ are constants such that $\alpha<\beta$, and $\gamma$ is a positive constant.

Lemma 2.2: Let the scalar function $h(x, \alpha, \beta, \gamma)$ be defined as

$$
h(x, \alpha, \beta, \gamma)=\frac{f(\tau)}{f(\tau)+\gamma f(1-\tau)} \text { with } \tau=\frac{x-\alpha}{\beta-\gamma},
$$

where

$$
f(\tau)=0 \text { if } \tau \leq 0, \text { and } f(\tau)=e^{-\frac{1}{\tau}} \text { if } \tau>0,
$$

with $\alpha$ and $\beta$ constants such that $\alpha<\beta$, and $\gamma$ a positive constant. Then $h(x, \alpha, \beta, \gamma)$ is a smooth step function.

Proof. See Appendix B. An alternative "symmetric" (i.e., $\gamma=1$ ) smooth step function is available in [28] but it requires a numerical integration. The introduction of the positive constant $\gamma$ in the smooth step function in Lemma

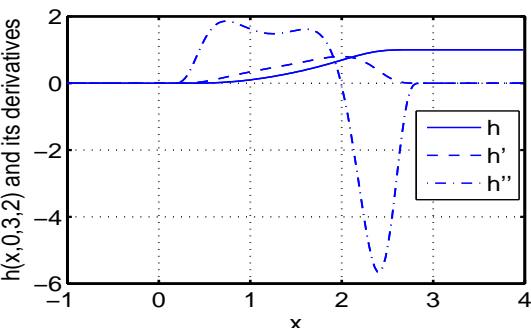

Fig. 3. A smooth step function and its first and second derivatives.
2.2 is to shift the location at which $h^{\prime}(x, \alpha, \beta, \gamma)$ attains its extremum value. An illustration of a smooth step function $(\alpha=0, \beta=3, \gamma=2)$ is given in Fig. 3

\section{Barbalat-like lemma}

The following Barbalat-like lemma is to be used in stability analysis of the closed loop system in Appendix C.

Lemma 2.3: Assume that a nonnegative scalar differentiable function $f(t)$ satisfies the following conditions

$$
\text { 1) } \left.\left|\frac{d}{d t} f(t)\right| \leq k_{1} f(t), \forall t \geq 0,2\right) \int_{0}^{\infty} f(t) d t \leq k_{2},
$$

where $k_{1}$ and $k_{2}$ are positive constants, then $\lim _{t \rightarrow \infty} f(t)=0$.

Proof. See [10]. Lemma 2.3 differs from Barbalat's lemma found in [29]. While Barbalat's lemma assumes that $f(t)$ is uniformly continuous, Lemma 2.3 assumes that $\left|\frac{d}{d t} f(t)\right|$ is bounded by $k_{1} f(t)$. Lemma 2.3 is useful in proving convergence of $f(t)$ when it is difficult to prove uniform continuity of $f(t)$.

\section{PROBlem Statement}

\section{A. Agent dynamics}

As mentioned before this paper mainly focuses on difficulties caused by the ellipsoidal shape of the agents in the coordination control design, we therefore assume that each ellipsoidal agent $i$ has the following dynamics:

$$
\begin{aligned}
& \dot{\boldsymbol{q}}_{i}=\boldsymbol{u}_{i}, \\
& \dot{\boldsymbol{\eta}}_{i}=\boldsymbol{\omega}_{i}, \quad i \in \mathbb{N},
\end{aligned}
$$

where $\mathbb{N}$ is the set of all agents in the group, $\boldsymbol{u}_{i}=$ $\left[\begin{array}{lll}u_{x i} & u_{y i} & u_{z i}\end{array}\right]^{T}$ and $\boldsymbol{\omega}_{i}=\left[\begin{array}{lll}\omega_{\phi i} & \omega_{\theta i} & \omega_{\psi i}\end{array}\right]^{T}$ are the control input vectors of the agent $i$. It is recalled that $\boldsymbol{q}_{i}=\left[\begin{array}{lll}x_{i} & y_{i} & z_{i}\end{array}\right]^{T}$ with $\left(x_{i}, y_{i}, z_{i}\right)$ being the position coordinates of the center of the agent $i$ and $\boldsymbol{\eta}_{i}=\left[\phi_{i} \theta_{i} \psi_{i}\right]^{T}$ with $\left(\phi_{i}, \theta_{i}, \psi_{i}\right)$ being the orientation angles of the agent $i$, see Fig. 2. For agents with higher order dynamics, the backstepping technique [30] can be used because we will design the control input vectors $\boldsymbol{u}_{i}$ and $\boldsymbol{\omega}_{i}$ such that they are smooth. 


\section{B. Coordination control objective}

In order to design a coordination control system for a group of ellipsoidal agents, there is a need of specifying a common goal for the group, sensing capacity of the agents, and initial position and orientation of the agents. We therefore impose the following assumption on the reference trajectory, sensing capacity of each agent, and initial conditions between the agents.

Assumption 3.1:

1) The reference position and orientation vectors $\boldsymbol{q}_{i d}(t)$ and $\boldsymbol{\eta}_{i d}(t)$ for the agent $i$ to track satisfy the condition:

$$
\Delta_{i j d}(t) \geq \delta_{i j d}, \forall(i, j) \in \mathbb{N}, j \neq i, t \geq 0,
$$

where $\delta_{i j d}$ is a positive constant. The term $\Delta_{i j d}(t)$ is $\Delta_{i j}(t)$ given in (1) with $\boldsymbol{q}_{i}(t), \boldsymbol{q}_{j}(t), \boldsymbol{\eta}_{i}(t)$, and $\boldsymbol{\eta}_{j}(t)$ replaced by $\boldsymbol{q}_{i d}(t), \boldsymbol{q}_{j d}(t), \boldsymbol{\eta}_{i d}(t)$, and $\boldsymbol{\eta}_{j d}(t)$, respectively. Moreover, $\left\|\dot{\boldsymbol{q}}_{i d}(t)\right\|,\left\|\dot{\boldsymbol{q}}_{i d}(t)\right\|$, and $\left\|\boldsymbol{q}_{i j d}(t)\right\|$ with $\boldsymbol{q}_{i j d}(t)=\boldsymbol{q}_{i d}(t)-$ $\boldsymbol{q}_{j d}(t)$ are bounded for all $(i, j) \in \mathbb{N}, j \neq j$, and $t \geq 0$.

2) The agents $i$ and $j$ have spherical sensing spaces, which are centered at the points $O_{i}$ and $O_{j}$, and have radii of $R_{i}$ and $R_{j}$, respectively. The radii $R_{i}$ and $R_{j}$ are sufficiently large in the sense that

$$
\Delta_{i j R}^{m}>0
$$

where $\Delta_{i j R}^{m}$ is the greatest lower bound of $\Delta_{i j}$ when the agents $i$ and $j$ are within their sensing ranges, i.e.,

$$
\Delta_{i j R}^{m}=\inf \left(\Delta_{i j}\right) \text { s.t. }\left\{\begin{array}{l}
\boldsymbol{\eta}_{i j} \in \mathbb{R}^{3}, \\
\left\|\boldsymbol{q}_{i j}\right\|=\min \left(R_{i}, R_{j}\right),
\end{array}\right.
$$

for all $(i, j) \in \mathbb{N}$ and $j \neq i$.

3 ) The agent $i$ can sense the states, $\boldsymbol{q}_{j}$ and $\boldsymbol{\eta}_{j}$, of the agent $j$ if the agent $j$ is inside the sensing space of the agent $i$.

4) At the initial time $t_{0} \geq 0$, all the agents in the group are sufficiently far away from each other in the sense that the following condition holds:

$$
\Delta_{i j}\left(t_{0}\right)>0,
$$

where $\Delta_{i j}\left(t_{0}\right)$ is given in (1) evaluated at $\left(\boldsymbol{q}_{i}=\boldsymbol{q}_{i}\left(t_{0}\right), \boldsymbol{\eta}_{i}=\right.$ $\left.\boldsymbol{\eta}_{i}\left(t_{0}\right)\right)$ and $\left(\boldsymbol{q}_{j}=\boldsymbol{q}_{j}\left(t_{0}\right), \boldsymbol{\eta}_{j}=\boldsymbol{\eta}_{j}\left(t_{0}\right)\right)$, and we have abused the notation of $\Delta_{i j}\left(\boldsymbol{q}_{i j}\left(t_{0}\right), \boldsymbol{\eta}_{i}\left(t_{0}\right), \boldsymbol{\eta}_{j}\left(t_{0}\right)\right)$ as $\Delta_{i j}\left(t_{0}\right)$ for simplicity of presentation.

Remark 3.1: 1) Assumption 3.1.1 specifies feasible reference trajectories $\boldsymbol{q}_{i d}(t)$ and $\boldsymbol{\eta}_{i d}(t)$ for the agent $i$ in the group to track since they have to satisfy the condition (14). A desired coordination shape can be specified by the reference trajectories $\boldsymbol{q}_{i d}(t)$ and $\boldsymbol{\eta}_{i d}(t)$ with $i=1, \ldots, N$. Let us consider the virtual structure approach in [8], [9], [10] to generate the reference trajectories $\boldsymbol{q}_{i d}(t)$ and $\boldsymbol{\eta}_{i d}(t)$ for the agent $i$ to track. First, a virtual structure consisting of $N$ vertices is designed as a desired coordination shape. Second, we let the center of the virtual structure move along a predefined trajectory (often called the common reference trajectory). Third, as the virtual structure moves, its vertex $i$ generates the reference trajectories $\boldsymbol{q}_{i d}(t)$ and $\boldsymbol{\eta}_{i d}(t)$ for the agent $i$ to track. Several examples can be found in [10].

2) In Assumption 3.1.2, the condition (15) holds if there exists a positive constant $\varrho_{i}$ such that $R_{i} \geq \varrho_{i}+\sup \left(a_{i}+\right.$ $\left.a_{j}, a_{i}+b_{j}, a_{i}+c_{j}, b_{i}+a_{j}, b_{i}+b_{j}, b_{i}+c_{j}, c_{i}+a_{j}, c_{i}+b_{j}, c_{i}+c_{j}\right)$, for all $(i, j) \in \mathbb{N}$ and $j \neq i$.

Coordination Control Objective 3.1: Under Assumption 3.1 , for each agent $i$ design the control input vectors $\boldsymbol{u}_{i}$ and $\boldsymbol{\omega}_{i}$ such that the position and orientation vectors $\left(\boldsymbol{q}_{i}, \boldsymbol{\eta}_{i}\right)$ of the agent $i$ track its reference position and orientation vectors $\left(\boldsymbol{q}_{i d}, \boldsymbol{\eta}_{i d}\right)$ while avoiding collision with all other agents in the group. Specifically, we will design $\boldsymbol{u}_{i}$ and $\boldsymbol{\omega}_{i}$ such that

$$
\begin{aligned}
& \lim _{t \rightarrow \infty} \chi_{i e}(t)=0, \\
& \Delta_{i j}(t) \geq \delta_{i j}, \quad \forall t \geq t_{0} \geq 0,
\end{aligned}
$$

where $\chi_{i e}(t)=\left(\left(\boldsymbol{q}_{i}(t)-\boldsymbol{q}_{i d}(t)\right),\left(\boldsymbol{\eta}_{i}(t)-\boldsymbol{\eta}_{i d}(t)\right)\right)$, for all $(i, j) \in \mathbb{N}, i \neq j$, and $t \geq t_{0} \geq 0$, where $\delta_{i j}$ is a positive constant.

\section{Coordination Control Design}

\section{A. Pairwise Collision Avoidance Functions}

1) Construction of Pairwise Collision Avoidance Functions: This section defines and constructs pairwise collision avoidance functions that will be used in a potential function for the coordination control design.

Definition 4.1: Let $\varphi_{i j}$ be a scalar function of the transformed distance $\Delta_{i j}$ given in (1) of the ellipsoidal agents $i$ and $j$. The function $\varphi_{i j}$ is said to be a pairwise collision avoidance function if it has the following properties:

$$
\begin{aligned}
& \text { 1) } \varphi_{i j}=0, \varphi_{i j}^{\prime}=0, \varphi_{i j}^{\prime \prime}=0, \forall \Delta_{i j} \in\left[\Delta_{i j}^{*}, \infty\right) \text {, } \\
& \text { 2) } \varphi_{i j}>0, \forall \Delta_{i j} \in\left(0, \Delta_{i j}^{*}\right), \\
& \text { 3) } \lim _{\Delta_{i j} \rightarrow 0} \varphi_{i j}=\infty, \lim _{\Delta_{i j} \rightarrow 0} \varphi_{i j}^{\prime}=-\infty, \\
& \text { 4) } \varphi_{i j} \text { is smooth, } \forall \Delta_{i j} \in(0, \infty),
\end{aligned}
$$

where $\varphi_{i j}^{\prime}=\frac{\partial \varphi_{i j}}{\partial \Delta_{i j}}$ and $\varphi_{i j}^{\prime \prime}=\frac{\partial^{2} \varphi_{i j}}{\partial \Delta_{i j}^{2}}$. The positive constant $\Delta_{i j}^{*}$ is referred to as the effective collision avoidance transformed distance between the agents $i$ and $j$, and satisfies the condition

$$
0<\Delta_{i j}^{*}<\min \left(\Delta_{i j R}^{m}, \delta_{i j d}\right),
$$

with $\Delta_{i j R}^{m}$ defined in (16).

Remark 4.1: Property 1) implies that the function $\varphi_{i j}$ is zero when the agents $i$ and $j$ are outside of their communication ranges since the constant $\Delta_{i j}^{*}$ satisfies the condition (20). Property 2) implies that the function $\varphi_{i j}$ is positive definite when the agents $i$ and $j$ are inside of their communication ranges. By Lemma 2.1, Property 3) means that the function $\varphi_{i j}$ is equal to infinity when a collision between the agents $i$ and $j$ occurs. Property 4) allows us to use control design and stability analysis methods found in [29] for continuous systems instead of techniques for switched and discontinuous systems found in [31] to handle the collision avoidance problem under the agents' limited communication ranges.

Lemma 4.1: Let the scalar function $\varphi_{i j}$ be defined as

$$
\varphi_{i j}=\frac{1-h\left(\Delta_{i j}, \alpha_{i j}, \beta_{i j}, \gamma_{i j}\right)}{\Delta_{i j}}
$$

where the positive constants $\alpha_{i j}$ and $\beta_{i j}$ satisfy the condition

$$
0<\alpha_{i j}<\beta_{i j} \leq \Delta_{i j}^{*}
$$


and $\gamma_{i j}$ is a positive constant. The function $h\left(\Delta_{i j}, \alpha_{i j}, \beta_{i j}, \gamma_{i j}\right)$ is a smooth step function defined in Definition 2.1.

Then the function $\varphi_{i j}$ is a pairwise collision avoidance function.

Proof. From (21), we have

$$
\begin{aligned}
\varphi_{i j}^{\prime} & =-\frac{h^{\prime}(\bullet)}{\Delta_{i j}}-\frac{1-h(\bullet)}{\Delta_{i j}^{2}} \\
\varphi_{i j}^{\prime \prime} & =-\frac{h^{\prime \prime}(\bullet)}{\Delta_{i j}}+\frac{2 h^{\prime}(\bullet)}{\Delta_{i j}^{2}}+\frac{2(1-h(\bullet))}{\Delta_{i j}^{3}}
\end{aligned}
$$

where • stands for $\left(\Delta_{i j}, \alpha_{i j}, \beta_{i j}, \gamma_{i j}\right)$. From (21) and (23), it is trivial to show that the function $\varphi_{i j}$ holds all properties listed in (19) by using properties of the smooth step function $h\left(\Delta_{i j}, \alpha_{i j}, \beta_{i j}, \gamma_{i j}\right)$ listed in (9) with a note that the constants $\alpha_{i j}$ and $\beta_{i j}$ satisfy the condition (22). A pairwise collision avoidance function $\varphi_{i j}$ with $\alpha_{i j}=0.5, \beta_{i j}=2$, and $\gamma_{i j}=1$ is plotted in Fig. 4.

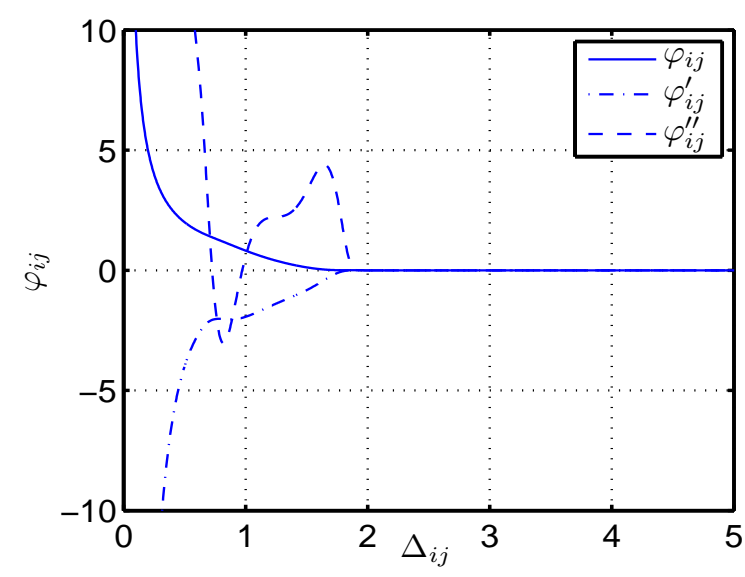

Fig. 4. A pairwise collision avoidance function and its derivatives.

2) Derivative of Pairwise Collision Avoidance Functions: To prepare for the control design later, we calculate the derivative of the pairwise collision avoidance function $\varphi_{i j}$. As such, it is noted $\Delta_{i j}$ is a smooth function of $\boldsymbol{q}_{i j}, \boldsymbol{\eta}_{i}$, and $\boldsymbol{\eta}_{i j}$, see Remark 2.1. However, there is a difficulty in determining an explicit dependence of $\Delta_{i j}$ on $\boldsymbol{q}_{i j}, \boldsymbol{\eta}_{i}$, and $\boldsymbol{\eta}_{i j}$ via the matrix $\boldsymbol{Q}_{i j}$, see (1) and (2), because $\kappa_{i j}$ cannot be solved explicitly. To avoid this difficulty, we treat $\Delta_{i j}$ as a smooth function of $\kappa_{i j}, \overline{\boldsymbol{q}}_{i j}$, and $\boldsymbol{\eta}_{i j}$.

We first calculate the first time derivative of $\kappa_{i j}$. From (7), we have

$$
\dot{\kappa}_{i j}=-\left(\frac{\partial F_{i j}}{\partial \kappa_{i j}}\right)^{-1}\left(\left(\frac{\partial F_{i j}}{\partial \overline{\boldsymbol{q}}_{i j}}\right)^{T} \dot{\overline{\boldsymbol{q}}}_{i j}+\left(\frac{\partial F_{i j}}{\partial \boldsymbol{\eta}_{i j}}\right)^{T} \dot{\boldsymbol{\eta}}_{i j}\right)
$$

where

$F_{i j}\left(\kappa_{i j}\right)=\overline{\boldsymbol{q}}_{i j}^{T}\left(\boldsymbol{I}_{3 \times 3}+\kappa_{i j} \boldsymbol{T}_{j}\right)^{-T} \boldsymbol{T}_{j}\left(\boldsymbol{I}_{3 \times 3}+\kappa_{i j} \boldsymbol{T}_{j}\right)^{-1} \overline{\boldsymbol{q}}_{i j}-1$.

It is noted that $\frac{\partial F_{i j}}{\partial \kappa_{i j}}$ is always nonzero, see Subsection A-3. Hence, the first time derivative of $\Delta_{i j}$ is

$$
\dot{\Delta}_{i j}=\boldsymbol{G}_{i j} \dot{\overline{\boldsymbol{q}}}_{i j}+\boldsymbol{H}_{i j} \dot{\boldsymbol{\eta}}_{i j}
$$

where

$$
\begin{aligned}
& \boldsymbol{G}_{i j}=\left[\frac{\partial \Delta_{i j}}{\partial \overline{\boldsymbol{q}}_{i j}}-\frac{\partial \Delta_{i j}}{\partial \kappa_{i j}}\left(\frac{\partial F_{i j}}{\partial \kappa_{i j}}\right)^{-1} \frac{\partial F_{i j}}{\partial \overline{\boldsymbol{q}}_{i j}}\right]^{T}, \\
& \boldsymbol{H}_{i j}=\left[\frac{\partial \Delta_{i j}}{\partial \boldsymbol{\eta}_{i j}}-\frac{\partial \Delta_{i j}}{\partial \kappa_{i j}}\left(\frac{\partial F_{i j}}{\partial \kappa_{i j}}\right)^{-1} \frac{\partial F_{i j}}{\partial \boldsymbol{\eta}_{i j}}\right]^{T} .
\end{aligned}
$$

From definition of $\overline{\boldsymbol{q}}_{i j}$ in (2), we have

$$
\dot{\overline{\boldsymbol{q}}}_{i j}=\boldsymbol{P}_{i} \dot{\boldsymbol{q}}_{i j}+\boldsymbol{S}_{i} \dot{\boldsymbol{\eta}}_{i}
$$

where the matrix $S_{i}$ is defined by

$$
\dot{\boldsymbol{P}}_{i} \boldsymbol{q}_{i j}=\boldsymbol{S}_{i} \dot{\boldsymbol{\eta}}_{i}
$$

Substituting (28) into (26) results in

$$
\dot{\Delta}_{i j}=\boldsymbol{G}_{i j} \boldsymbol{P}_{i} \dot{\boldsymbol{q}}_{i j}+\boldsymbol{G}_{i j} \boldsymbol{S}_{i} \dot{\boldsymbol{\eta}}_{i}+\boldsymbol{H}_{i j} \dot{\boldsymbol{\eta}}_{i j} .
$$

Remark 4.2: The transformed distance $\Delta_{i j}$ depends not only on the relative distance vector $\boldsymbol{q}_{i j}$ and the relative orientation vector $\boldsymbol{\eta}_{i j}$ but also on the individual orientation vector $\boldsymbol{\eta}_{i}$ of the ellipsoidal agent $i$. This dependence creates a difficulty in designing control algorithms using a gradient based approach in the sense that it is hard to write the derivative of the pairwise collision avoidance function $\varphi_{i j}$ as a product of the gradient of individual agents $i$ and $j$ and their state derivatives. Later, this difficulty will be overcome by a special design of the virtual reference trajectories with the use of a smooth step function.

With (30), the derivative of the pairwise collision avoidance function $\varphi_{i j}$ is given by

$$
\dot{\varphi}_{i j}=\varphi_{i j}^{\prime}\left(\boldsymbol{G}_{i j} \boldsymbol{P}_{i} \dot{\boldsymbol{q}}_{i j}+\boldsymbol{G}_{i j} \boldsymbol{S}_{i} \dot{\boldsymbol{\eta}}_{i}+\boldsymbol{H}_{i j} \dot{\boldsymbol{\eta}}_{i j}\right), \forall \Delta_{i j}>0 \text {. }
$$

The condition $\Delta_{i j}>0$ will be guaranteed by our control design later.

\section{B. Lyapunov Function}

Since the derivative of the pairwise collision avoidance function $\varphi_{i j}$ is the summation of the term $\varphi_{i j}^{\prime} \boldsymbol{G}_{i j} \boldsymbol{P}_{i} \dot{\boldsymbol{q}}_{i j}$, $\varphi_{i j}^{\prime} \boldsymbol{G}_{i j} \boldsymbol{S}_{i} \dot{\boldsymbol{\eta}}_{i}$, and $\varphi_{i j}^{\prime} \boldsymbol{H}_{i j} \dot{\boldsymbol{\eta}}_{i j}$ instead of the summation of $\varphi_{i j}^{\prime} \boldsymbol{G}_{i j} \boldsymbol{P}_{i}\left(\dot{\boldsymbol{q}}_{i j}-\dot{\boldsymbol{q}}_{i j d}\right), \varphi_{i j}^{\prime} \boldsymbol{G}_{i j} \boldsymbol{S}_{i}\left(\dot{\boldsymbol{\eta}}_{i}-\dot{\boldsymbol{\eta}}_{i d}\right)$, and $\varphi_{i j}^{\prime} \boldsymbol{H}_{i j}\left(\dot{\boldsymbol{\eta}}_{i j}-\right.$ $\dot{\boldsymbol{\eta}}_{i j d}$ ) with $\dot{\boldsymbol{\eta}}_{i j d}=\dot{\boldsymbol{\eta}}_{i d}-\dot{\boldsymbol{\eta}}_{j d}$, it is not possible to use a Lyapunov function candidate as a summation of all the pairwise collision functions and the square of all the errors $\boldsymbol{q}_{i}-\boldsymbol{q}_{i d}$ and $\boldsymbol{\eta}_{i}-\boldsymbol{\eta}_{i d}$ for the coordination control design, see also Remark 4.2.

To overcome the aforementioned impossibilities, we will construct a Lyapunov function candidate as a sum of all the pairwise collision avoidance functions $\varphi_{i j}$ in (21) and the square of all the errors $\left(\boldsymbol{q}_{i}-\boldsymbol{q}_{i d}^{*}\right)$ and $\left(\boldsymbol{\eta}_{i}-\boldsymbol{\eta}_{i d}^{*}\right)$. The vectors $\boldsymbol{q}_{i d}^{*}$ and $\boldsymbol{\eta}_{i d}^{*}$ are considered as the virtual reference trajectories to be designed such that $\lim _{t \rightarrow \infty} \boldsymbol{q}_{i d}^{*}(t)=\boldsymbol{q}_{i d}(t)$ and $\lim _{t \rightarrow \infty} \boldsymbol{\eta}_{i d}^{*}(t)=\boldsymbol{\eta}_{i d}(t)$. As such, the Lyapunov function candidate for the coordination control design in the next section is constructed as follows

$$
\begin{aligned}
V= & \sum_{i=1}^{N-1} \sum_{j=i+1}^{N} \varphi_{i j}+\frac{1}{2} \sum_{i=1}^{N}\left(\left(\boldsymbol{q}_{i}-\boldsymbol{q}_{i d}^{*}\right)^{T} \boldsymbol{C}_{1}\left(\boldsymbol{q}_{i}-\boldsymbol{q}_{i d}^{*}\right)+\right. \\
& \left.\left(\boldsymbol{\eta}_{i}-\boldsymbol{\eta}_{i d}^{*}\right)^{T} \boldsymbol{C}_{2}\left(\boldsymbol{\eta}_{i}-\boldsymbol{\eta}_{i d}^{*}\right)\right),
\end{aligned}
$$


where $C_{1}$ and $C_{2}$ are symmetric positive definite matrices. Differentiating both sides of (32) along the solutions of (31) and recalling from (13) that $\dot{\boldsymbol{q}}_{i}=\boldsymbol{u}_{i}$ and $\dot{\boldsymbol{\eta}}_{i}=\boldsymbol{\omega}_{i}$ result in

$$
\begin{aligned}
\dot{V}= & \sum_{i=1}^{N}\left(\left[\boldsymbol{\Omega}_{i}^{T}\left(\boldsymbol{u}_{i}-\dot{\boldsymbol{q}}_{i d}^{*}\right)+\boldsymbol{\Xi}_{i}^{T}\left(\boldsymbol{\omega}_{i}-\dot{\boldsymbol{\eta}}_{i d}^{*}\right)\right]+\right. \\
& {\left.\left[\boldsymbol{\Psi}_{1 i}^{T} \dot{\boldsymbol{q}}_{i d}^{*}+\boldsymbol{\Psi}_{2 i}^{T} \dot{\boldsymbol{\eta}}_{i d}^{*}\right]\right), }
\end{aligned}
$$

where we added and subtracted $\varphi_{i j}^{\prime} \boldsymbol{G}_{i j} \boldsymbol{P}_{i} \dot{\boldsymbol{q}}_{i j d}, \varphi_{i j}^{\prime}\left(\boldsymbol{G}_{i j} \boldsymbol{S}_{i}+\right.$ $\left.\boldsymbol{H}_{i j}\right) \dot{\boldsymbol{\eta}}_{i d}^{*}$, and $-\varphi_{i j}^{\prime} \boldsymbol{H}_{i j} \dot{\boldsymbol{\eta}}_{j d}^{*}$ to the right hand side of the equation (31) before substituting this equation into the derivative of $V$. In (33), we have defined

$$
\begin{aligned}
& \boldsymbol{\Omega}_{i}=\left[\boldsymbol{\Psi}_{1 i}^{T}+\boldsymbol{C}_{1}\left(\boldsymbol{q}_{i}-\boldsymbol{q}_{i d}^{*}\right)\right]^{T}, \\
& \boldsymbol{\Xi}_{i}=\left[\boldsymbol{\Psi}_{2 i}^{T}+\boldsymbol{C}_{2}\left(\boldsymbol{\eta}_{i}-\boldsymbol{\eta}_{i d}^{*}\right)\right]^{T}, \\
& \left.\boldsymbol{\Psi}_{1 i}=\left[-\sum_{j=1}^{i-1} \varphi_{j i}^{\prime} \boldsymbol{G}_{j i} \boldsymbol{P}_{j}+\sum_{j=i+1}^{N} \varphi_{i j}^{\prime} \boldsymbol{G}_{i j} \boldsymbol{P}_{i}\right)\right]^{T}, \\
& \boldsymbol{\Psi}_{2 i}=\left[-\sum_{j=1}^{i-1} \varphi_{j i}^{\prime} \boldsymbol{H}_{j i}+\sum_{j=i+1}^{N} \varphi_{i j}^{\prime}\left(\boldsymbol{G}_{i j} \boldsymbol{S}_{i}+\boldsymbol{H}_{i j}\right)\right]^{T} .
\end{aligned}
$$

\section{Control law}

We first deal with the terms inside the first square bracket in the right hand side of (33). As such, to avoid a large control effort when an agent in the group is close to the agent $i$ due to Property 3) of the function $\varphi_{i j}$, see (19), for collision avoidance, we design a control law for $\boldsymbol{u}_{i}$ and $\boldsymbol{\omega}_{i}$ as follows:

$$
\begin{aligned}
\boldsymbol{u}_{i} & =-\boldsymbol{K}_{1} \boldsymbol{W}\left(\boldsymbol{\Omega}_{i}\right)+\dot{\boldsymbol{q}}_{i d}^{*}, \\
\boldsymbol{\omega}_{i} & =-\boldsymbol{K}_{2} \boldsymbol{W}\left(\boldsymbol{\Xi}_{i}\right)+\dot{\boldsymbol{\eta}}_{i d}^{*},
\end{aligned}
$$

where $\boldsymbol{K}_{1}$ and $\boldsymbol{K}_{2}$ are symmetric positive definite matrices. The vector $\boldsymbol{W}(\boldsymbol{\chi})$ denotes a vector of bounded functions of elements of $\chi$ in the sense that $\boldsymbol{W}(\boldsymbol{\chi})=$ $\left[w\left(\chi_{1}\right) \ldots, w\left(\chi_{l}\right), \ldots, w\left(\chi_{n}\right)\right]^{T}$ with $\chi_{l}$ the $l^{\text {th }}$ element of $\chi$, i.e., $\chi=\left[\chi_{1} \ldots, \chi_{l}, \ldots, \chi_{n}\right]^{T}$. The function $w(\chi)$ is a scalar, differentiable and bounded function, and satisfies

1) $|w(\chi)| \leq M_{1}$,

2) $w(\chi)=0 \quad$ if $\chi=0, \quad \chi w(\chi)>0$ if $\chi \neq 0$,

3) $w(-\chi)=-w(\chi),(\chi-\omega)[w(\chi)-w(\omega)] \geq 0$,

4) $\left|\frac{w(\chi)}{\chi}\right| \leq M_{2},\left|\frac{\partial w(\chi)}{\partial \chi}\right| \leq M_{3},\left.\frac{\partial w(\chi)}{\partial \chi}\right|_{\chi=0}=1$,

for all $\chi \in \mathbb{R}, \omega \in \mathbb{R}$, where $M_{1}, M_{2}, M_{3}$ are positive constants. Some functions that satisfy the above properties are $\arctan (\chi)$ and $\tanh (\chi)$.

We now deal with the second square bracket in the right hand side of (33). The terms inside this square bracket seem to be troublesome because $\dot{\boldsymbol{q}}_{i d}^{*}$ and $\dot{\boldsymbol{\eta}}_{i d}^{*}$ are nonzero in general since we are solving the coordination tracking control problem. Moreover, we require that $\boldsymbol{\eta}_{i d}^{*}$ and $\boldsymbol{\eta}_{i d}^{*}$ asymptotically tend to the reference trajectories $\boldsymbol{q}_{i d}$ and $\boldsymbol{\eta}_{i d}$, respectively. To get around these problems, we will design update laws $\dot{\boldsymbol{q}}_{i d}^{*}$ and $\dot{\boldsymbol{\eta}}_{i d}^{*}$ such that

$$
\boldsymbol{\Psi}_{1 i}^{T} \dot{\boldsymbol{q}}_{i d}^{*}=0, \boldsymbol{\Psi}_{2 i}^{T} \dot{\boldsymbol{\eta}}_{i d}^{*}=0,
$$

hold for all time and such that $\boldsymbol{\eta}_{i d}^{*}$ and $\boldsymbol{\eta}_{i d}^{*}$ asymptotically tend to $\boldsymbol{q}_{i d}$ and $\boldsymbol{\eta}_{i d}$, respectively. As such, we utilize smooth step functions to design update laws $\dot{\boldsymbol{q}}_{i d}^{*}$ and $\dot{\boldsymbol{\eta}}_{i d}^{*}$ as follows:

$$
\begin{aligned}
& \dot{\boldsymbol{q}}_{i d}^{*}=\left[\prod_{j \neq i} h\left(\Delta_{i j}, \alpha_{i j d}, \beta_{i j d}, \gamma_{i j d}\right)\right]\left(-\boldsymbol{K}_{1 d}\left(\boldsymbol{q}_{i d}^{*}-\boldsymbol{q}_{i d}\right)+\dot{\boldsymbol{q}}_{i d}\right), \\
& \boldsymbol{q}_{i d}^{*}\left(t_{0}\right)=\boldsymbol{q}_{i d}\left(t_{0}\right), \\
& \dot{\boldsymbol{\eta}}_{i d}^{*}=\left[\prod_{j \neq i} h\left(\Delta_{i j}, \alpha_{i j d}, \beta_{i j d}, \gamma_{i j d}\right)\right]\left(-\boldsymbol{K}_{2 d}\left(\boldsymbol{\eta}_{i d}^{*}-\boldsymbol{\eta}_{i d}\right)+\dot{\boldsymbol{\eta}}_{i d}\right), \\
& \boldsymbol{\eta}_{i d}^{*}\left(t_{0}\right)=\boldsymbol{\eta}_{i d}\left(t_{0}\right),
\end{aligned}
$$

where $\boldsymbol{K}_{1 d}$ and $\boldsymbol{K}_{2 d}$ are symmetric positive definite matrices. The function $h\left(\Delta_{i j}, \alpha_{i j d}, \beta_{i j d}, \gamma_{i j d}\right)$ is a smooth step function with the constants $\alpha_{i j d}, \beta_{i j d}$, and $\gamma_{i j d}$ chosen as:

$$
\alpha_{i j d}=\beta_{i j}, \alpha_{i j d}<\beta_{i j d} \leq \Delta_{i j}^{*}, \gamma_{i j d}>0,
$$

where $b_{i j}$ is chosen as in (22), and $\Delta_{i j}^{*}$ satisfies the condition (20). Using properties of the smooth step function, the choice of the constants $\alpha_{i j}, \beta_{i j}, \alpha_{i j d}$ and $\beta_{i j d}$ in (22) and (39) results in $h^{\prime}\left(\Delta_{i j}, \alpha_{i j}, \beta_{i j}, \gamma_{i j}\right) h\left(\Delta_{i j}, \alpha_{i j d}, \beta_{i j d}, \gamma_{i j d}\right)=0$ and $\left(1-h\left(\Delta_{i j}, \alpha_{i j}, \beta_{i j}, \gamma_{i j}\right)\right) h\left(\Delta_{i j}, \alpha_{i j d}, \beta_{i j d}, \gamma_{i j d}\right)=0$. These equalities imply that (37) holds as long as $\Delta_{i j}>0$, which is to be guaranteed by our control design. Moreover, the choice of the constants $\alpha_{i j d}$ and $\beta_{i j d}$ in (39) ensures that the function $h\left(\Delta_{i j}, \alpha_{i j d}, \beta_{i j d}, \gamma_{i j d}\right)$ approaches 1 whenever $\Delta_{i j}$ approaches a value less than $\beta_{i j}$. Also, it is recalled from (22) that $\beta_{i j}<\min \left(\Delta_{i j R}^{m}, \delta_{i j d}\right.$. Therefore, the choice of update laws in (38) achieves our purpose: $\boldsymbol{\eta}_{i d}^{*}$ and $\boldsymbol{\eta}_{i d}^{*}$ asymptotically tend to the reference trajectories $\boldsymbol{q}_{i d}$ and $\boldsymbol{\eta}_{i d}$, respectively, as long as $\lim _{t \rightarrow \infty} \Delta_{i j}(t)<\beta_{i j}$. This limit and the inequality $\Delta_{i j}>0$ will be guaranteed by our designed control input vectors $\boldsymbol{u}_{i}$ and $\boldsymbol{\omega}_{i}$ in (35). This will be shown in the proof of the main result.

Remark 4.3: 1) The control vectors $\boldsymbol{u}_{i}$ and $\boldsymbol{\omega}_{i}$ in (35) of the agent $i$ are smooth and depend on only its own state and the reference trajectory, and the states of other agents $j$ in the communication range of the agent $i$ due to Property 1 ) of the pairwise collision avoidance function $\varphi_{i j}$ in (19).

2) The update laws $\dot{\boldsymbol{q}}_{i d}^{*}$ and $\dot{\boldsymbol{\eta}}_{i d}^{*}$ in (38) ensure that when the collision avoidance is active, the virtual reference trajectories $\boldsymbol{q}_{i d}^{*}$ and $\boldsymbol{\eta}_{i d}^{*}$ are not updated. This implies that the control vectors $\boldsymbol{u}_{i}$ and $\boldsymbol{\omega}_{i}$ give priority to the collision avoidance mission or the reference trajectory tracking mission whenever which mission is more important.

Substituting the control vectors $\boldsymbol{u}_{i}$ and $\boldsymbol{\omega}_{i}$ in (35) and the update laws $\dot{\boldsymbol{q}}_{i d}^{*}$ and $\dot{\boldsymbol{\eta}}_{i d}^{*}$ in (38) into (33) results in

$$
\dot{V}=-\sum_{i=1}^{N} \vartheta_{i}
$$

where

$$
\vartheta_{i}=\boldsymbol{\Omega}_{i}^{T} \boldsymbol{K}_{1} \boldsymbol{W}\left(\boldsymbol{\Omega}_{i}\right)+\boldsymbol{\Xi}_{i}^{T} \boldsymbol{K}_{2} \boldsymbol{W}\left(\boldsymbol{\Xi}_{i}\right)
$$

On the other hand, substituting the control vectors $\boldsymbol{u}_{i}$ and $\boldsymbol{\omega}_{i}$ in (35) and the update laws $\dot{\boldsymbol{q}}_{i d}^{*}$ and $\dot{\boldsymbol{\eta}}_{i d}^{*}$ in (38) into (13) results 
in the closed loop system:

$$
\begin{aligned}
& \dot{\boldsymbol{q}}_{i}=-\boldsymbol{K}_{1} \boldsymbol{W}\left(\boldsymbol{\Omega}_{i}\right)+\dot{\boldsymbol{q}}_{i d}^{*}, \\
& \dot{\boldsymbol{\eta}}_{i}=-\boldsymbol{K}_{2} \boldsymbol{W}\left(\boldsymbol{\Xi}_{i}\right)+\dot{\boldsymbol{\eta}}_{i d}^{*},
\end{aligned}
$$

for all $i \in \mathbb{N}$. We now present the main result of our paper in the following theorem.

Theorem 4.1: Under Assumption 3.1, the smooth control vectors $\boldsymbol{u}_{i}$ and $\boldsymbol{\omega}_{i}$ in (35) and the update laws $\dot{\boldsymbol{q}}_{i d}^{*}$ and $\dot{\boldsymbol{\eta}}_{i d}^{*}$ in (38) for the agent $i$ solve the coordination control objective as long as the control design parameters $\boldsymbol{K}_{1}, \boldsymbol{K}_{2}, \boldsymbol{K}_{1 d}, \boldsymbol{K}_{2 d}$, $\boldsymbol{C}_{1}, \boldsymbol{C}_{2}, \alpha_{i j}, \beta_{i j}, \alpha_{i j d}$, and $\beta_{i j d}$ are chosen such that the conditions (14), (15), (17), (22) and (39) hold. In particular, no collisions between any agents can occur for all $t \geq t_{0} \geq$ 0 , the closed loop system (42) is forward complete, and the trajectories $\boldsymbol{q}_{i}$ and $\boldsymbol{\eta}_{i}$ of the agent $i$ asymptotically track its reference trajectories $\boldsymbol{q}_{i d}$ and $\boldsymbol{\eta}_{i d}$, respectively, for all $i=$ $1, \ldots, N$.

Proof. See Appendix C.

\section{Simulation Results}

In this section, we provide a numerical simulation to illustrate the effectiveness of the proposed coordination control design stated in Theorem 4.1. We use $N=6$ ellipsoidal agents with the geometric parameters as $a_{i}=3$ and $b_{i}=c_{i}=1$ for all $i=1, \ldots, N$. The initial position and orientation of these agents are chosen as follows:

$$
\begin{aligned}
& \boldsymbol{q}_{i}(0)=-R_{0} \boldsymbol{L}_{i}, \\
& \boldsymbol{\eta}_{i}(0)=\left[\begin{array}{lll}
0 & 0 & 0
\end{array}\right], \forall i \in \mathbb{N},
\end{aligned}
$$

where $R_{0}=9$, and

$$
\begin{aligned}
& \boldsymbol{L}_{1}=\left[\begin{array}{lll}
1 & 0 & 0
\end{array}\right], \boldsymbol{L}_{2}=\left[\begin{array}{lll}
0 & 1 & 0
\end{array}\right], \boldsymbol{L}_{3}=\left[\begin{array}{lll}
-1 & 0 & 0
\end{array}\right], \\
& \boldsymbol{L}_{4}=\left[\begin{array}{lll}
0 & -1 & 0
\end{array}\right], \boldsymbol{L}_{5}=\left[\begin{array}{lll}
0 & 0 & 1
\end{array}\right], \boldsymbol{L}_{6}=\left[\begin{array}{lll}
0 & 0 & -1
\end{array}\right]
\end{aligned}
$$

The above choice of initial conditions means that all the agents are uniformly distributed on a sphere, which is centered at the origin and has a radius of $R_{0}$. All the agents have the same sensing range with $R_{i}=25$.

The reference trajectories are chosen as

$$
\begin{aligned}
& \boldsymbol{q}_{i d}=R_{0} \boldsymbol{L}_{i}+\boldsymbol{q}_{\text {od }}, \\
& \boldsymbol{\eta}_{i d}=[0 \pi / 4 \pi / 4], \forall i \in \mathbb{N},
\end{aligned}
$$

where the common reference trajectory $\boldsymbol{q}_{o d}$ is generated by $\dot{\boldsymbol{q}}_{\text {od }}=\left[\begin{array}{lll}1 & 1 & 1\end{array}\right]^{T}$ with $\boldsymbol{q}_{\text {od }}(0)=[0,0,0]^{T}$. This choice implies that the reference trajectories are angled straight lines, and that the agents are to be uniformly distributed on a sphere, which is centered at the common reference trajectory $\boldsymbol{q}_{o d}$ and has a radius of $R_{0}$.

The control design parameters are chosen as follows: $\boldsymbol{K}_{1}=$ $\boldsymbol{K}_{2}=\operatorname{diag}(20,20,20), \boldsymbol{K}_{1 d}=\boldsymbol{K}_{2 d}=\operatorname{diag}(2,2,2), \boldsymbol{C}_{1}=$ $\boldsymbol{C}_{2}=\operatorname{diag}(50,50,50), \alpha_{i j}=5, \beta_{i j}=1.5 \alpha_{i j}, \alpha_{i j d}=\beta_{i j}$, $\beta_{i j d}=1.2 \alpha_{i j d}$. The function $w(\cdot)$ is chosen as $\arctan (\cdot)$.

A calculation shows that the above initial conditions and the above choice of control design parameters satisfy all the conditions (14), (15), (17), (22), (39).

Simulation results are plotted in Fig.5, Fig. 6, and Fig. 7. In Fig.5, several snapshots of the position and orientation of all agents are plotted. The representative $\Delta_{i j}^{*}=\left(\Pi_{j \in \mathbb{N} j \neq i} \Delta_{i j}\right)^{1 / 5}$ is plotted in the first sub-figure of Fig.6. The control inputs $\boldsymbol{u}=\left[\boldsymbol{u}_{1}, \ldots, \boldsymbol{u}_{i}, \ldots, \boldsymbol{u}_{N}\right]^{T}$ and $\boldsymbol{\omega}=\left[\boldsymbol{\omega}_{1}, \ldots, \boldsymbol{\omega}_{i}, \ldots, \boldsymbol{\omega}_{N}\right]^{T}$ are plotted in the second and third sub-figures of Fig.6. It is clearly seen from Fig.5 and Fig.6 that there is no collision between any agents as indicated by $\Delta_{i j}^{*}>0$ for all $i \in \mathbb{N}$ even though the above choice of initial conditions and reference trajectories makes the space around the origin "crowded" since all the agents need to cross this space to track their reference trajectories. Moreover, all the agents manage to track their reference trajectories asymptotically as seen from the tracking errors $\boldsymbol{q}-\boldsymbol{q}_{d}=\left[\boldsymbol{q}_{1}-\boldsymbol{q}_{1 d}, \ldots, \boldsymbol{q}_{i}-\boldsymbol{q}_{i d}, \ldots, \boldsymbol{q}_{N}-\boldsymbol{q}_{N d}\right]^{T}$ and $\boldsymbol{\eta}-\boldsymbol{\eta}_{d}=\left[\boldsymbol{\eta}_{1}-\boldsymbol{\eta}_{1 d}, \ldots, \boldsymbol{\eta}_{i}-\boldsymbol{\eta}_{i d}, \ldots, \boldsymbol{\eta}_{N}-\boldsymbol{\eta}_{N d}\right]^{T}$ in Fig.7.

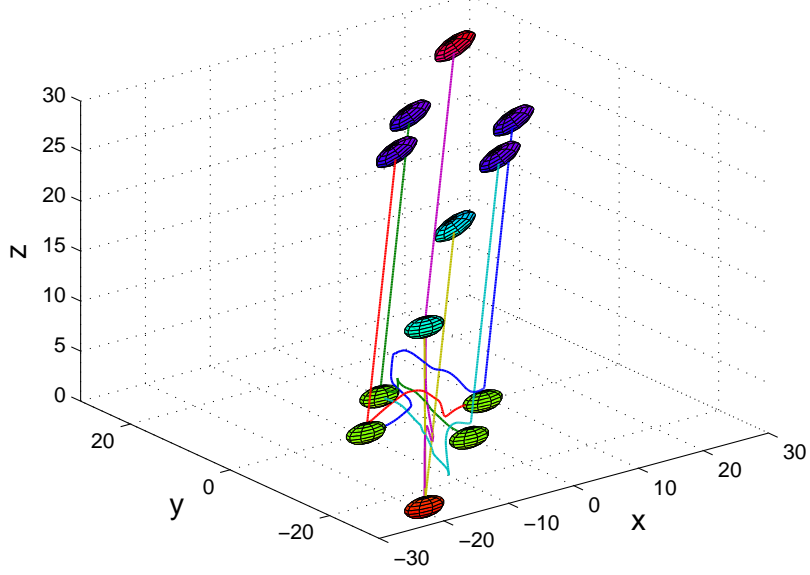

Fig. 5. Snapshots of the agents' position and orientation.
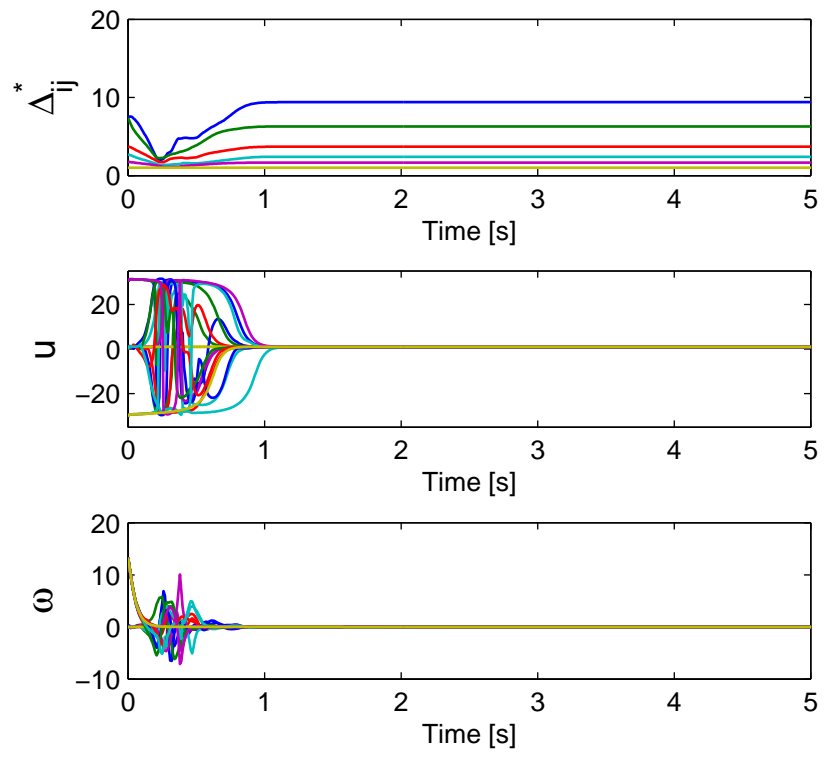

Fig. 6. Representative $\Delta_{i j}^{*}$ and control inputs. 

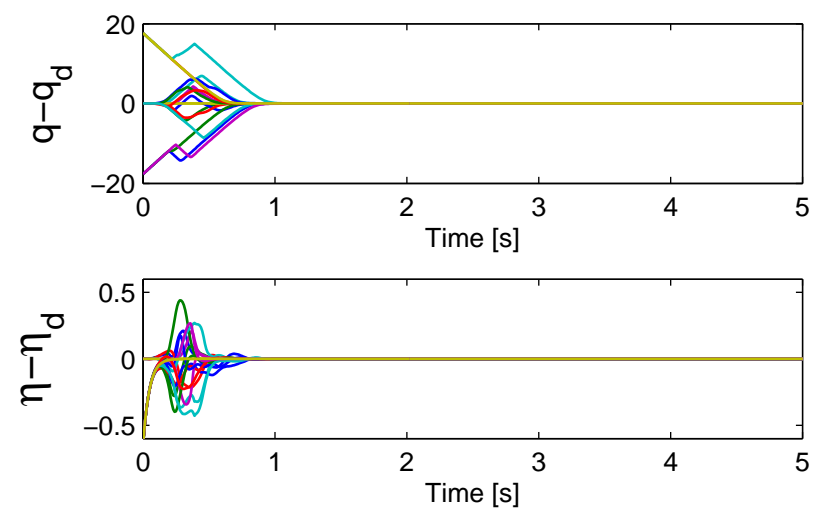

Fig. 7. Tracking errors.

\section{CONClusions}

This paper has presented a constructive method to design a coordination control system for ellipsoidal agents. The tools used for the success of the coordination control design included a separation condition between two ellipsoids, smooth step functions, and novel pairwise collision avoidance functions. An extension of the proposed coordination control design in this paper and those controllers designed for single underactuated underwater vehicles in [32] to provide to a coordination control system for a group of underactuated underwater vehicles is under consideration.

\section{APPENDIX A}

\section{PROOF OF LEMMA 2.1}

From Fig. 2, the boundaries of the ellipsoids $i$ and $j$ (equations of the points $w_{i b}$ and $w_{j b}$ ) coordinated in the $O_{b i} X_{b i} Y_{b i} Z_{b i}$ frame attached to the ellipsoid $i$ can be described by

$$
\begin{aligned}
& E_{i}: \boldsymbol{q}_{i b}^{T} \boldsymbol{A}_{i}^{-2} \boldsymbol{q}_{i b}=1, \\
& E_{j}: \boldsymbol{q}_{j b}=-\boldsymbol{R}^{-1}\left(\boldsymbol{\eta}_{i}\right) \boldsymbol{q}_{i j}+\boldsymbol{R}^{-1}\left(\boldsymbol{\eta}_{i j}\right) \boldsymbol{A}_{j} \boldsymbol{\varrho}_{j},
\end{aligned}
$$

where $\boldsymbol{A}_{i}, \boldsymbol{A}_{j}, \boldsymbol{q}_{i j}$, and $\boldsymbol{\eta}_{i j}$ are defined in (3) and (6); and $\varrho_{j}=\left[\cos \left(\alpha_{j}\right) \cos \left(\beta_{j}\right) \cos \left(\alpha_{j}\right) \sin \left(\beta_{j}\right) \sin \left(\alpha_{j}\right)\right]^{T}$ with $\alpha_{j} \in\left[-\frac{\pi}{2}, \frac{\pi}{2}\right]$ and $\beta_{j} \in[-\pi, \pi]$ are auxiliary angles; and $\boldsymbol{q}_{i b}$ and $\boldsymbol{q}_{j b}$ are vectors denoting positions of the points $w_{i b}$ and $w_{j b}$, respectively. The idea to prove Lemma 2.1 consists of two steps: 1) transforming the ellipsoids $i$ and $j$ to a unit sphere and an ellipsoid; 2) calculating the distance between the transformed sphere and the transformed ellipsoid.

1) Transformation: We transform the ellipsoids $i$ and $j$ to a unit sphere and an ellipsoid by the following coordinate transformation:

$$
\begin{gathered}
\overline{\boldsymbol{q}}_{i b}=\boldsymbol{A}_{i}^{-1}\left(\boldsymbol{q}_{i b}+\boldsymbol{R}^{-1}\left(\boldsymbol{\eta}_{i}\right) \boldsymbol{q}_{i j}\right), \\
\overline{\boldsymbol{q}}_{j b}=\boldsymbol{A}_{i}^{-1}\left(\boldsymbol{q}_{j b}+\boldsymbol{R}^{-1}\left(\boldsymbol{\eta}_{i}\right) \boldsymbol{q}_{i j}\right) .
\end{gathered}
$$

With the above coordinate transformation, the ellipsoids (46) are transformed to a unit sphere and an ellipsoid as follows:

$$
\begin{aligned}
& \bar{E}_{i}:\left(\overline{\boldsymbol{q}}_{i b}+\overline{\boldsymbol{q}}_{i j}\right)^{T}\left(\overline{\boldsymbol{q}}_{i b}+\overline{\boldsymbol{q}}_{i j}\right)=1, \\
& \bar{E}_{j}: \overline{\boldsymbol{q}}_{j b}=\boldsymbol{A}_{i}^{-1} \boldsymbol{R}\left(\boldsymbol{\eta}_{i j}\right) \boldsymbol{A}_{j} \varrho_{j} .
\end{aligned}
$$

Now, the ellipsoid $E_{i}$ has become the unit sphere $\bar{E}_{i}$ centered at the point $O_{u s i}$ whose coordinates are described by the first equation in (48). The ellipsoid $E_{j}$ has become another ellipsoid $\bar{E}_{j}$ centered at the origin of the $O_{b i} X_{b i} Y_{b i} Z_{b i}$ frame, i.e., the point $O_{b i}$.

For convenience of calculating the distance between the unit sphere $\bar{E}_{i}$ and the ellipsoid $\bar{E}_{j}$, we will rewrite the ellipsoid $\bar{E}_{j}$ in an implicit form instead of parametric form given in the second equation of (48). By squaring both sides of each row of $\overline{\boldsymbol{q}}_{j b}=\boldsymbol{A}_{i}^{-1} \boldsymbol{R}\left(\boldsymbol{\eta}_{i j}\right) \boldsymbol{A}_{j} \boldsymbol{\varrho}_{j}$ then adding the results together, we have $\overline{\boldsymbol{q}}_{j b}^{T} \boldsymbol{T}_{j} \overline{\boldsymbol{q}}_{j b}=1$ where $\boldsymbol{T}_{j}$ is defined in (4). Hence, the unit sphere and the ellipsoid defined in (48) can be rewritten as

$$
\begin{aligned}
& \bar{E}_{i}:\left(\overline{\boldsymbol{q}}_{i b}+\overline{\boldsymbol{q}}_{i j}\right)^{T}\left(\overline{\boldsymbol{q}}_{i b}+\overline{\boldsymbol{q}}_{i j}\right)=1, \\
& \bar{E}_{j}: \overline{\boldsymbol{q}}_{j b}^{T} \boldsymbol{T}_{j} \overline{\boldsymbol{q}}_{j b}=1 .
\end{aligned}
$$

2) Distance $\Delta_{i j}$ : We now calculate the distance from the center of the unit sphere $\bar{E}_{i}$ described by the first equation in (49), i.e., from the point $O_{u s i}$ to the ellipsoid $\bar{E}_{j}$ described by the second equation in (49). A necessary condition for a point $\overline{\boldsymbol{q}}_{j b}$ to be the closest point to the point $O_{u s i}$ is that $\overline{\boldsymbol{q}}_{i j}-\overline{\boldsymbol{q}}_{j b}$ is perpendicular to the tangent plane to the ellipsoid $\bar{E}_{j}$ at $\overline{\boldsymbol{q}}_{j b}$. Since the surface gradient $\frac{\partial f\left(\overline{\boldsymbol{q}}_{j b}\right)}{\partial \overline{\boldsymbol{q}}_{j b}}$ with $f\left(\overline{\boldsymbol{q}}_{j b}\right):=\frac{1}{2}\left(\overline{\boldsymbol{q}}_{j b}^{T} \boldsymbol{T}_{j} \overline{\boldsymbol{q}}_{j b}-1\right)$ is normal to the ellipsoid's surface, the algebraic condition for the closest point $\left.\overline{\boldsymbol{q}}_{i b}\right)$ is

$$
\overline{\boldsymbol{q}}_{i j}-\overline{\boldsymbol{q}}_{j b}=\kappa_{i j} \frac{\partial f\left(\overline{\boldsymbol{q}}_{j b}\right)}{\partial \overline{\boldsymbol{q}}_{j b}} .
$$

For the point $O_{u s i}$ outside the ellipsoid $\bar{E}_{j}$, there is only one point on the ellipsoid whose normal points toward the point $O_{u s i}$. However, there can be as many as five other points whose surface normals point directly away from $O_{u s i}$. The point on the ellipsoid whose normal points toward the point $O_{u s i}$ corresponds to the largest root $\kappa_{i j}$ of the equation (50). Moreover, $\overline{\boldsymbol{q}}_{j b}$ must satisfy the ellipsoid equation, i.e., the second equation of (49).

From (50), we have

$$
\overline{\boldsymbol{q}}_{j b}=\left(\boldsymbol{I}_{3 \times 3}+\kappa_{i j} \boldsymbol{T}_{j}\right)^{-1} \overline{\boldsymbol{q}}_{i j},
$$

which is substituted into the second equation in (49) results in the equation (7).

Now the distance from the point $O_{u s i}$ to the closest point $\overline{\boldsymbol{q}}_{j b}$ on the ellipsoid $\bar{E}_{j}$ described by the second equation in (49) is given by

$$
\Delta_{i j}=\left\|\overline{\boldsymbol{q}}_{i j}-\overline{\boldsymbol{q}}_{j b}\right\|-1,
$$

where $\overline{\boldsymbol{q}}_{j b}$ is the solution of (50) and the second equation of (49) with $\kappa_{i j}$ being the largest root. Substituting $\overline{\boldsymbol{q}}_{j b}$ in (51) and $\overline{\boldsymbol{q}}_{i j}$ in (2) into (52) results in (1).

It is noted that after $\overline{\boldsymbol{q}}_{j b}$ is found, we can determine the intersection point with coordinates $\overline{\boldsymbol{q}}_{i b}$ on the unit sphere $\bar{E}_{i}$ between the line from the point $O_{u s i}$ to the point with coordinates $\overline{\boldsymbol{q}}_{j b}$ and the unit sphere $\bar{E}_{i}$. Once we obtain the coordinates $\overline{\boldsymbol{q}}_{i b}$ and $\overline{\boldsymbol{q}}_{j b}$ with respect to the closest distance between the unit sphere $\bar{E}_{i}$ and the transformed ellipsoid $\bar{E}_{j}$, the corresponding coordinates $\boldsymbol{q}_{i b}$ and $\boldsymbol{q}_{j b}$ with respect to the shortest distance between the original ellipsoids $E_{i}$ 
and $E_{j}$ on the original ellipsoids $E_{i}$ and $E_{j}$ can be directly determined from (47). The actual distance between the original ellipsoids $E_{i}$ and $E_{j}$ is $\left\|\boldsymbol{q}_{i b}-\boldsymbol{q}_{j b}\right\|$. For a coordination control application, the transformed distance $\Delta_{i j}$ is sufficient because from the transformation (47) we can see that $\Delta_{i j}>0$ implies that $\left\|\boldsymbol{q}_{i b}-\boldsymbol{q}_{j b}\right\|>0$ and vice versa.

Therefore, two ellipsoids $i$ and $j$ are separated if $\Delta_{i j}>0$, i.e., the condition (8) must hold.

3) Solution of equations (7): We first show that the equation (7) has a unique root on the domain of interest. Let us define $\kappa_{i j}^{L}$ be the largest root of the equation $\operatorname{det}\left(\boldsymbol{I}_{3 \times 3}+\kappa_{i j} \boldsymbol{T}_{j}\right)=0$. This is a cubic equation and can be solved for its roots explicitly. We now observe that for any nonzero $\overline{\boldsymbol{q}}_{i j}$, i.e., $\left\|\overline{\boldsymbol{q}}_{i j}\right\|>0$, the following inequalities and limits hold:

$$
\begin{aligned}
& \frac{\partial F\left(\kappa_{i j}\right)}{\partial \kappa_{i j}}<0, \frac{\partial^{2} F\left(\kappa_{i j}\right)}{\partial \kappa_{i j}^{2}}>0, \quad \forall \kappa_{i j} \in\left(\kappa_{i j}^{L}, \infty\right), \\
& \lim _{\kappa_{i j} \rightarrow \kappa_{i j}^{L}} F_{i j}\left(\kappa_{i j}\right)=\infty, \lim _{\kappa_{i j} \rightarrow \infty} F_{i j}\left(\kappa_{i j}\right)=-1,
\end{aligned}
$$

because the matrix $\boldsymbol{T}_{j}$ is symmetric and positive definite with its elements given in (5). Properties of $F_{i j}\left(\kappa_{i j}\right)$ in (53) imply that the function $F_{i j}\left(\kappa_{i j}\right)$ is strictly decreasing from $\infty$ to -1 on the domain $\kappa_{i j} \in\left(\kappa_{i j}^{L}, \infty\right)$. Therefore, the equation (7) has a unique root on the domain of interest. Moreover, this root is also the largest root of (7).

Given an initial value $\kappa_{i j}(0)=\kappa_{i j}^{L}+\epsilon$ where $\epsilon$ is a positive constant such that $F_{i j}\left(\kappa_{i j}^{L}+\epsilon\right)>0$, a numerical procedure using the Newton method to calculate the largest root $\kappa_{i j}$ is given as follows [33]:

$$
\kappa_{i j}(n+1)=\kappa_{i j}(n)-\frac{F_{i j}\left(\kappa_{i j}(n)\right)}{F_{i j}^{\prime}\left(\kappa_{i j}(n)\right)},
$$

where $F_{i j}^{\prime}\left(\kappa_{i j}(n)\right)=\left.\frac{\partial F_{i j}\left(\kappa_{i j}\right)}{\partial \kappa_{i j}}\right|_{\kappa_{i j}=\kappa_{i j}(n)}$ and $F_{i j}\left(\kappa_{i j}(n)\right)=$ $\left.F_{i j}\left(\kappa_{i j}\right)\right|_{\kappa_{i j}=\kappa_{i j}(n)}$ with $F_{i j}\left(\kappa_{i j}\right)$ given in (7). The algorithm (54) provides a quadratic convergence of $\kappa_{i j}(n)$ to the largest root $\kappa_{i j}$ of the equation (7), since $\frac{\partial F\left(\kappa_{i j}\right)}{\partial \kappa_{i j}}$ is nonzero and $\frac{\partial^{2} F\left(\kappa_{i j}\right)}{\partial \kappa_{i j}^{2}}$ is bounded in the domain of interest, see Theorem 1.1 in [33] for a proof. Indeed, after the largest root $\kappa_{i j}$ is found, $\overline{\boldsymbol{q}}_{j b}$ is obtained from (51). Proof of Lemma 2.1 is completed.

\section{APPENDIX B}

\section{PROOF OF LEMMA 2.2}

We need to verify that the function $h(x, \alpha, \beta, \gamma)$ given in (10) satisfies all properties defined in (9). To prove Property $1)$, we note from (10) that for all $-\infty<x \leq \alpha$, we have $\tau \leq 0$ and $1-\tau>0$. Hence by (11), we have $f(\tau)=0$ and $f(1-\tau)>0$, which are substituted into (10) to yield Property 1). Similarly, proof of Property 2 ) follows by noting from (10) that for all $\beta \leq x<\infty$, we have $\tau>0$ and $1-\tau \leq 0$. Hence by (11), we have $f(\tau)>0$ and $f(1-\tau)=0$, which are substituted into (10) to yield Property 2). Property 3 ) holds since for all $\alpha<x<\beta$ we have from (10) that $0<\tau<1$. Hence $f(\tau)>0$ and $f(1-\tau)>0$ by (11). Therefore, we have $0<\frac{f(\tau)}{f(\tau)+\gamma f(1-\tau)}<1$ for all $\alpha<x<\beta$ and $\gamma>0$. To prove Property 4 ), it is first noted that by definition of the function $f(\tau)$, see (11), $f(\tau)+\gamma f(1-\tau)>0$ for all $\tau \in \mathbb{R}$ since $\gamma>0$. In addition, the interval $\alpha<x<\beta$ is equivalent to the interval $0<\tau<1$, see (10). Therefore, the function $h(x, \alpha, \beta, \gamma)$ or $h(\tau, 0,1, \gamma)$ is infinite times differentiable for all $x \in \mathbb{R}$ or $\tau \in \mathbb{R}$. Next, we need to show that $\frac{\partial^{k} h(\tau, 0,1, \gamma)}{\partial \tau^{k}}$ tends to zero when $\tau$ tends to $0^{-}$or $1^{+}$for an arbitrary positive integer $k$, since $\frac{\partial^{k} h(\tau, 0,1, \gamma)}{\partial \tau^{k}}=0$ for all $\tau \leq 0$ or $1 \leq \tau$. For $0<\tau<1$, we can write the function $h(\tau, 0,1, \gamma)$ as

$$
h(\tau, 0,1, \gamma)=\frac{1}{1+\gamma e^{\xi}},
$$

where $\xi=\frac{1-2 \tau}{\tau(1-\tau)}$. From (55), we have

$$
\begin{aligned}
\frac{\partial h(\tau, 0,1, \gamma)}{\partial \xi} & =-\frac{\gamma e^{\xi}}{\left(1+\gamma e^{\xi}\right)^{2}}=-\frac{1}{1+\gamma e^{\xi}}+\frac{1}{\left(1+\gamma e^{\xi}\right)^{2}} \\
& =-h(\tau, 0,1, \gamma)+h^{2}(\tau, 0,1, \gamma) .
\end{aligned}
$$

Using (56), a calculation shows that

$$
\begin{array}{r}
\frac{\partial^{k} h(\tau, 0,1, \gamma)}{\partial \tau^{k}}=\left(P_{1}\left(\frac{1}{\tau}, \frac{1}{\tau-1}\right)+P_{2}\left(\frac{1}{\tau}, \frac{1}{\tau-1}\right) \times\right. \\
Q(h(\tau, 0,1, \gamma)))\left(-h(\tau, 0,1, \gamma)+h^{2}(\tau, 0,1, \gamma)\right),
\end{array}
$$

where $P_{i}\left(\frac{1}{\tau}, \frac{1}{\tau-1}\right), i=1,2$ are $2 k$-order polynomials of $\frac{1}{\tau}$ and $\frac{1}{1-\tau}$, and $Q(h(\tau, 0,1, \gamma))$ is a $k-1$-order polynomial of $h(\tau, 0,1, \gamma)$. Since $\lim _{x \rightarrow 0^{-}} \frac{e^{-\frac{1}{x}}}{x^{m}}=0$ for any positive integer $m$ and $Q(h(\tau, 0,1, \gamma))$ is bounded by some constant (since $0<h(\tau, 0,1, \gamma)<1)$, it can be directly deduce from (57) the limits $\lim _{\tau \rightarrow 0^{-}} \frac{\partial^{k} h(\tau, 0,1, \gamma)}{\partial \tau^{k}}=0$ and $\lim _{\tau \rightarrow 1^{+}} \frac{\partial^{k} h(\tau, 0,1, \gamma)}{\partial \tau^{k}, \gamma}=$ 0. Property 5) holds since $\frac{\partial h(x, \alpha, \beta, \gamma)}{\partial x}=\frac{\partial h(\tau, 0,1, \gamma)}{\partial \tau} \frac{\partial \tau}{\partial x}=$ $\left(-h(\tau, 0,1, \gamma)+h^{2}(\tau, 0,1, \gamma)\right) \frac{\partial \xi}{\partial \tau} \frac{\partial \tau}{\partial x}$ with $\frac{\partial \tau}{\partial x}=\frac{\partial \tau}{\beta-\alpha}$, $\frac{\partial \xi}{\partial \tau}=-\frac{1}{\tau^{2}}-\frac{1}{(\tau-1)^{2}}$, and $\left(-h(\tau, 0,1, \gamma)+h^{2}(\tau, 0,1, \gamma)\right)<0$ for all $\tau \in(0,1)$. To prove Property 6), using (57) we calculate $\frac{\partial^{2} h(x, \alpha, \beta, \gamma)}{\partial x^{2}}$ as follows

$$
\begin{gathered}
\frac{\partial^{2} h(x, \alpha, \beta, \gamma)}{\partial x^{2}}=\left(-h(x, \alpha, \beta, \gamma)+h^{2}(x, \alpha, \beta, \gamma)\right) \times \\
\left(\xi^{\prime \prime}+(1-2 h(x, \alpha, \beta, \gamma)) \xi^{\prime 2}\right) \frac{\partial \tau}{\partial x},
\end{gathered}
$$

where $\xi^{\prime}=\frac{\partial \xi}{\partial \tau}=-\frac{1}{\tau^{2}}-\frac{1}{(\tau-1)^{2}}$, and $\xi^{\prime \prime}=\frac{\partial^{2} \xi}{\partial \tau^{2}}=$ $\frac{2}{\tau^{3}}+\frac{2}{(\tau-1)^{3}}$. For a $x^{*} \in(a, b)$, we have $\left(-h\left(x^{*}, \alpha, \beta, \gamma\right)+\right.$ $\left.h^{2}\left(x^{*}, \alpha, \beta, \gamma\right)\right)<0$. Using this information and setting $\left.\frac{\partial^{2} h(x, \alpha, \beta, \gamma)}{\partial x^{2}}\right|_{x=x^{*}}=0$, we have from (58) that

$$
\gamma=\left.e^{-\xi} \frac{\xi^{2}+\xi^{\prime \prime}}{\xi^{\prime 2}-\xi^{\prime \prime}}\right|_{x=x^{*}} .
$$

We now need to show that $\gamma$ is always positive for all $x^{*} \in$ $(\alpha, \beta)$. This is sufficient to to prove that $\xi^{\prime 2}+\xi^{\prime \prime}>0$ and $\xi^{\prime 2}-\xi^{\prime \prime}>0$ for all $\tau \in(0,1)$. As such, after an algebraic 
calculation from expressions of $\xi^{\prime \prime}$ and $\xi^{\prime}$ we have

$$
\begin{aligned}
\xi^{\prime 2}+\xi^{\prime \prime} & =\frac{1}{\tau^{4}}+\frac{2}{\tau^{3}}+\frac{2}{\tau^{2}(\tau-1)^{2}}+\frac{\tau}{(\tau-1)^{4}}, \\
\xi^{\prime 2}-\xi^{\prime \prime} & =\frac{1}{\tau^{4}(\tau-1)^{2}}\left(2 \tau^{2}(1-\tau)+(2 \tau-1)^{2}\right)+ \\
& \frac{1}{\tau^{2}(\tau-1)^{4}}\left(2 \tau^{2}(1-\tau)+\tau^{2}+(\tau-1)^{2}\right),
\end{aligned}
$$

which clearly imply that $\xi^{\prime 2}+\xi^{\prime \prime}>0$ and $\xi^{\prime 2}-\xi^{\prime \prime}>0$ for all $\tau \in(0,1)$.

\section{APPENDIX C}

\section{ProOF OF THEOREM 4.1}

A. Proof of no collisions and complete forwardness of the closed loop system

It is seen from (40) that $\dot{V} \leq 0$. Integrating $\dot{V} \leq 0$ from $t_{0}$ to $t$ and using the definition of $V$ in (32) with $\varphi_{i j}$ in (21) result in

$$
V(t) \leq V\left(t_{0}\right)
$$

where

$$
\begin{array}{r}
V(t)=\sum_{i=1}^{N-1} \sum_{j=i+1}^{N} \varphi_{i j}(t)+\frac{1}{2} \sum_{i=1}^{N}\left(\left(\boldsymbol{q}_{i}(t)-\boldsymbol{q}_{i d}^{*}(t)\right)^{T} \boldsymbol{C}_{1} \times\right. \\
\left.\left(\boldsymbol{q}_{i}(t)-\boldsymbol{q}_{i d}^{*}(t)\right)+\left(\boldsymbol{\eta}_{i}(t)-\boldsymbol{\eta}_{i d}^{*}(t)\right)^{T} \boldsymbol{C}_{2}\left(\boldsymbol{\eta}_{i}(t)-\boldsymbol{\eta}_{i d}^{*}(t)\right)\right),
\end{array}
$$

and $V\left(t_{0}\right)$ is $V(t)$ with $t$ replaced by $t_{0}$, for all $t \geq t_{0} \geq 0$. From the condition specified in item 4) of Assumption 3.1, Property 3) of $\varphi_{i j}$, and the initial values $\boldsymbol{q}_{i d}^{*}\left(t_{0}\right)$ and $\boldsymbol{\eta}_{i d}^{*}\left(t_{0}\right)$ in (38), we have the right hand side of (61) is bounded by a positive constant depending on the initial conditions. Boundedness of the right hand side of (61) implies that the left hand side of (61) must be also bounded. As a result, $\varphi_{i j}\left(\Delta_{i j}(t)\right)$ must be smaller than some positive constant depending on the initial conditions for all $t \geq t_{0} \geq 0$. From properties of $\varphi_{i j}$, see (19), $\Delta_{i j}(t)$, for all $(i, j) \in \mathbb{N}$ and $i \neq j$, must be larger than 0 for all $t \geq t_{0} \geq 0$. This in turn implies from Lemma 2.1 that there are no collisions between any agents for all $t \geq t_{0} \geq 0$. Boundedness of the left hand side of (61) also implies that of $\left(\boldsymbol{q}_{i}(t)-\boldsymbol{q}_{i d}^{*}(t)\right)$ and $\left(\boldsymbol{\eta}_{i}(t)-\boldsymbol{\eta}_{i d}^{*}(t)\right)$ for all $t \geq t_{0} \geq 0$. Since we have already proved that $\Delta_{i j}(t)>0$, the update laws $\dot{\boldsymbol{q}}_{i d}^{*}$ and $\dot{\boldsymbol{\eta}}_{i d}^{*}$ in (38) imply that $\left(\boldsymbol{q}_{i d}(t)-\boldsymbol{q}_{i d}^{*}(t)\right)$ and $\left(\boldsymbol{\eta}_{i d}(t)-\boldsymbol{\eta}_{i d}^{*}(t)\right)$ are also bounded. Therefore, the closed loop system (42) with the update laws (38) is forward complete.

\section{B. Equilibrium set}

We use Lemma 2.3 to find the equilibrium set, which the trajectories of the closed loop system (42) tend to. Integrating both sides of (40) gives $\int_{0}^{\infty} \vartheta(t) d t \leq V\left(t_{0}\right)$, where $\vartheta=$ $\sum_{i=1}^{N} \vartheta_{i}$ with $\vartheta_{i}$ defined in (41). The function $\vartheta(t)$ is scalar, nonnegative and differentiable. The derivative of $\vartheta(t)$ along the solutions of the closed loop system (42) using Properties 1), 2) and 4) of the function $\varphi_{i j}$ in (19) satisfies $\left|\frac{\mathrm{d} \vartheta(t)}{\mathrm{d} t}\right| \leq M \vartheta(t)$ with $M$ a positive constant. Therefore Lemma 2.3 results in $\lim _{t \rightarrow \infty} \vartheta(t)=0$, which means that $\lim _{t \rightarrow \infty} \vartheta_{i}(t)=0$. Therefore, from the expression of $\vartheta_{i}(t)$ in (41) and properties of the bounded function $\boldsymbol{W}(\bullet)$ in (36), we have

$$
\lim _{t \rightarrow \infty}\left(\boldsymbol{\Omega}_{i}(t), \boldsymbol{\Xi}_{i}(t)\right)=0 \text {. }
$$

Hence the trajectory $\left(\boldsymbol{q}_{i}, \boldsymbol{\eta}_{i}\right)$ of the agent $i$ asymptotically converges to the equilibrium set $\boldsymbol{E}$, in which $\boldsymbol{\Omega}_{i}(t)=0$ and $\Xi_{i}(t)=0$.

From the expression of $\boldsymbol{\Omega}_{i}$ and $\boldsymbol{\Xi}_{i}$, using properties of the pairwise collision avoidance function $\varphi_{i j}$ in (19) and the smooth step function $h\left(\Delta_{i j}, \alpha_{i j}, \beta_{i j}, \gamma_{i j}\right)$ in (9) with a note that the constants $\alpha_{i j}$ and $\beta_{i j}$ are chosen as in (22) and that the constants $\alpha_{i j d}$ and $\beta_{i j d}$ are chosen as in (39), the limits (63) imply that $\boldsymbol{\xi}=(\boldsymbol{q}, \boldsymbol{\eta})$, with $\boldsymbol{q}(t)=\left[\boldsymbol{q}_{1}^{T}(t) \boldsymbol{q}_{2}^{T}(t), \ldots, \boldsymbol{q}_{N}^{T}(t)\right]^{T}$ and $\boldsymbol{\eta}(t)=\left[\boldsymbol{\eta}_{1}^{T}(t) \boldsymbol{\eta}_{2}^{T}(t), \ldots, \boldsymbol{\eta}_{N}^{T}(t)\right]^{T}$, can tend to $\boldsymbol{\xi}_{d}=$ $\left(\boldsymbol{q}_{d}, \boldsymbol{\eta}_{d}\right)$, with $\boldsymbol{q}_{d}=\left[\boldsymbol{q}_{1 d}^{T} \boldsymbol{q}_{2 d}^{T}, \ldots, \boldsymbol{q}_{N d}^{T}\right]^{T}$ and $\boldsymbol{\eta}_{d}=$ $\left[\boldsymbol{\eta}_{1 d}^{T} \boldsymbol{\eta}_{2 d}^{T}, \ldots, \boldsymbol{\eta}_{N d}^{T}\right]^{T}$, since $\varphi_{i j}^{\prime}(t)=0$ at $\boldsymbol{q}_{i}=\boldsymbol{q}_{i d}, \boldsymbol{q}_{j}=\boldsymbol{q}_{j d}$, $\boldsymbol{\eta}_{i}=\boldsymbol{\eta}_{i d}$, and $\boldsymbol{\eta}_{j}=\boldsymbol{\eta}_{j d}$, for all $(i, j) \in \mathbb{N}$ and $i \neq j$, (see Property 1) of $\varphi_{i j}$ ), or tend to a vector denoted by $\boldsymbol{\xi}_{c}=\left(\boldsymbol{q}_{c}, \boldsymbol{\eta}_{c}\right)$, with $\boldsymbol{q}_{c}=\left[\boldsymbol{q}_{1 c}^{T} \boldsymbol{q}_{2 c}^{T}, \ldots, \boldsymbol{q}_{N c}^{T}\right]^{T}$ and $\boldsymbol{\eta}_{c}=$ $\left[\boldsymbol{\eta}_{1 c}^{T} \boldsymbol{\eta}_{2 c}^{T}, \ldots, \boldsymbol{\eta}_{N c}^{T}\right]^{T}$, as the time goes to infinity, i.e., the equilibrium sets can be $\boldsymbol{E}_{d}$ containing $\boldsymbol{\xi}_{d}$ or $\boldsymbol{E}_{c}$ containing $\boldsymbol{\xi}_{c}$. The vector $\boldsymbol{\xi}_{c}$ is such that

$$
\boldsymbol{\Omega}_{i c}=0, \boldsymbol{\Xi}_{i c}=0,
$$

where $\boldsymbol{\Omega}_{i c}=\left.\boldsymbol{\Omega}_{i}\right|_{\boldsymbol{\xi}=\boldsymbol{\xi}_{c}}$ and $\boldsymbol{\Xi}_{i c}=\left.\boldsymbol{\Xi}_{i}\right|_{\boldsymbol{\xi}=\boldsymbol{\xi}_{c}}$ for all $i \in \mathbb{N}$. Since we have already proved that the trajectory $\boldsymbol{\xi}$ can approach either the desired set $\boldsymbol{\Xi}_{d}$ of desired equilibrium points $\boldsymbol{\xi}_{d}$ or the undesired set $\boldsymbol{\Xi}_{c}$ of undesired equilibrium points $\boldsymbol{\xi}_{c}$ 'almost globally'. The term 'almost globally' refers to the fact that the agents start from a set that includes the condition (17) and that does not coincide at any point with the undesired set $\boldsymbol{\Xi}_{c}$. Hence, we need to prove that $\boldsymbol{\Xi}_{d}$ is locally asymptotically stable and that $\boldsymbol{\Xi}_{c}$ is locally unstable.

\section{Proof of $\boldsymbol{E}_{d}$ being asymptotically stable}

Linearizing the closed loop system (42) near $\boldsymbol{\xi}_{d}$ gives

$$
\begin{aligned}
& \dot{\boldsymbol{q}}_{i}=-\boldsymbol{K}_{1} \boldsymbol{C}_{1}\left(\boldsymbol{q}_{i}-\boldsymbol{q}_{i d}^{*}\right)+\dot{\boldsymbol{q}}_{i d}^{*}, \\
& \dot{\boldsymbol{\eta}}_{i}=-\boldsymbol{K}_{2} \boldsymbol{C}_{2}\left(\boldsymbol{\eta}_{i}-\boldsymbol{\eta}_{i d}^{*}\right)+\dot{\boldsymbol{q}}_{i d}^{*},
\end{aligned}
$$

for all $i \in \mathbb{N}$, where we have used $\left.\varphi_{i j}^{\prime}\right|_{\boldsymbol{\xi}=\boldsymbol{\xi}_{d}}=0$ and $\left.\varphi_{i j}^{\prime \prime}\right|_{\boldsymbol{\xi}=\boldsymbol{\xi}_{d}}=0$, see Property 1) of the function $\varphi_{i j}$ in (19), with a note that the constants $\alpha_{i j}$ and $\beta_{i j}$ are chosen as in (22) and that the constants $\alpha_{i j d}$ and $\beta_{i j d}$ are chosen as in (39). Moreover, linearizing the update laws (38) near $\boldsymbol{\xi}_{d}$ results in

$$
\begin{aligned}
& \dot{\boldsymbol{q}}_{i d}^{*}=-\boldsymbol{K}_{1 d}\left(\boldsymbol{q}_{i d}^{*}-\boldsymbol{q}_{i d}\right)+\dot{\boldsymbol{q}}_{i d}, \\
& \boldsymbol{q}_{i d}^{*}\left(t_{0}\right)=\boldsymbol{q}_{i d}\left(t_{0}\right), \\
& \dot{\boldsymbol{\eta}}_{i d}^{*}=-\boldsymbol{K}_{2 d}\left(\boldsymbol{\eta}_{i d}^{*}-\boldsymbol{\eta}_{i d}\right)+\dot{\boldsymbol{\eta}}_{i d}, \\
& \boldsymbol{\eta}_{i d}^{*}\left(t_{0}\right)=\boldsymbol{\eta}_{i d}\left(t_{0}\right),
\end{aligned}
$$

Local asymptotic stability of the equilibrium set $\boldsymbol{E}_{d}$ containing $\boldsymbol{\xi}_{d}$ follows from (65) and (66) because the first time derivative of the function $V_{d}=\frac{1}{2} \sum_{i=1}^{N}\left[\left\|\boldsymbol{\xi}_{i}-\boldsymbol{\xi}_{i d}^{*}\right\|^{2}+\left\|\boldsymbol{\xi}_{i d}-\boldsymbol{\xi}_{i d}^{*}\right\|^{2}\right]$ with $\boldsymbol{\xi}_{i d}=\left(\boldsymbol{q}_{i d}, \boldsymbol{\eta}_{i d}\right)$ and $\boldsymbol{\xi}_{i d}^{*}=\left(\boldsymbol{q}_{i d}^{*}, \boldsymbol{\eta}_{i d}^{*}\right)$ along the solutions of (65) and (66) satisfies $\dot{V}_{d} \leq-2 \min \left(\lambda_{\min }\left(\boldsymbol{K}_{1} \boldsymbol{C}_{1}\right)\right.$, $\left.\lambda_{\min }\left(\boldsymbol{K}_{2} \boldsymbol{C}_{2}\right), \lambda_{\min }\left(\boldsymbol{K}_{1 d}\right), \lambda_{\min }\left(\boldsymbol{K}_{2 d}\right)\right) V_{d}$ with $\lambda_{\min }(\bullet)$ being the minimum eigenvalue of the matrix $\bullet$. 


\section{Proof of $\boldsymbol{E}_{c}$ being unstable}

Substituting (64) into the closed loop system (42) gives

$$
\begin{aligned}
& \dot{\boldsymbol{q}}_{i}=-\boldsymbol{K}_{1}\left(\boldsymbol{W}\left(\boldsymbol{\Omega}_{i}\right)-\boldsymbol{W}\left(\boldsymbol{\Omega}_{i c}\right)\right)+\dot{\boldsymbol{q}}_{i d}^{*}, \\
& \dot{\boldsymbol{\eta}}_{i}=-\boldsymbol{K}_{2}\left(\boldsymbol{W}\left(\boldsymbol{\Xi}_{i}\right)-\boldsymbol{W}\left(\boldsymbol{\Xi}_{i c}\right)\right)+\dot{\boldsymbol{\eta}}_{i d}^{*},
\end{aligned}
$$

for all $i \in \mathbb{N}$, where we have used properties of $\boldsymbol{W}(\bullet)$ in (36) to have $\boldsymbol{W}\left(\boldsymbol{\Omega}_{i c}\right)=0$ and $\boldsymbol{W}\left(\boldsymbol{\Xi}_{i c}\right)=0$ from the equalities in (64). Since $\boldsymbol{\Omega}_{i c}=0$ and $\boldsymbol{\Xi}_{i c}=0$ at $\boldsymbol{\xi}_{c}$, using properties of $\boldsymbol{W}(\bullet)$ in (36) shows that local stability of (67) at $\boldsymbol{\xi}_{c}$ is equivalent to local stability of

$$
\begin{aligned}
& \dot{\boldsymbol{q}}_{i}=-\boldsymbol{K}_{1}\left(\boldsymbol{\Omega}_{i}-\boldsymbol{\Omega}_{i c}\right)+\dot{\boldsymbol{q}}_{i d}^{*}, \\
& \dot{\boldsymbol{\eta}}_{i}=-\boldsymbol{K}_{2}\left(\boldsymbol{\Xi}_{i}-\boldsymbol{\Xi}_{i c}\right)+\dot{\boldsymbol{\eta}}_{i d}^{*},
\end{aligned}
$$

at $\boldsymbol{\xi}_{c}$. We now investigate stability of (68) at $\boldsymbol{\xi}_{c}$.

Let $\mathbb{N}^{*}$ be the set of the agents such that if the agents $i$ and $j$ belong to the set $\mathbb{N}^{*}$ then $\Delta_{i j}\left(\boldsymbol{q}_{i j}, \boldsymbol{\eta}_{i}, \boldsymbol{\eta}_{i j}\right)<b_{i j}$ where it is recalled that $b_{i j}$ is chosen as in (22). Also let $N^{*}$ be the size of the set $\mathbb{N}^{*}$. For those agents in the set $\mathbb{N}^{*}$, the collision avoidance is active. Therefore, $\dot{\boldsymbol{\xi}}_{i d}^{*}=0$, for all $i \in \mathbb{N}^{*}$, see Item 2) in Remark 4.3. Let $\boldsymbol{q}_{i j c}=\boldsymbol{q}_{i c}-\boldsymbol{q}_{j c}, \boldsymbol{\eta}_{i j c}=\boldsymbol{\eta}_{i c}-\boldsymbol{\eta}_{j c}$, and $\varphi_{i j c}^{\prime}$ be $\varphi_{i j}^{\prime}$ evaluated at $\boldsymbol{q}_{i j}=\boldsymbol{q}_{i j c}, \boldsymbol{\eta}_{i}=\boldsymbol{\eta}_{i c}$, and $\boldsymbol{\eta}_{i j}=\boldsymbol{\eta}_{i j c}$. Now, from (64) we have

$$
\sum_{(i, j) \in \mathbb{N}^{*}} \boldsymbol{q}_{i j c}^{T} \boldsymbol{\Omega}_{i c}=0
$$

which can be expanded using (64) and (34) as follows:

$$
\sum_{(i, j) \in \mathbb{N}^{*}} \boldsymbol{q}_{i j c}^{T}\left(\boldsymbol{C}_{1}+N^{*} \varphi_{i j c}^{\prime} \boldsymbol{\Phi}_{i j c}\right) \boldsymbol{q}_{i j c}=\sum_{(i, j) \in \mathbb{N}^{*}} \boldsymbol{q}_{i j c}^{T} \boldsymbol{C}_{1} \boldsymbol{q}_{i j d}^{*}
$$

where the positive definite matrix $\boldsymbol{\Phi}_{i j c}$ depending on $\boldsymbol{q}_{i j c}, \boldsymbol{\eta}_{i c}$, and $\boldsymbol{\eta}_{i j c}$ is derived from (34), (27), and (1) such that (69) is equivalent to (70).

Since we have proved that $\left\|\boldsymbol{q}_{i j c}\right\|$ is bounded and $\left\|\boldsymbol{q}_{i j d}^{*}\right\|$ is bounded by the choice of update laws in (38) due to boundedness of $\left\|\boldsymbol{q}_{i j d}\right\|$ and $\left\|\dot{\boldsymbol{q}}_{i j d}\right\|$ by assumption, the equation (70) indicates that $\lim _{\lambda_{\max }\left(\boldsymbol{C}_{1}\right) \rightarrow 0}\left(\left\|\boldsymbol{Q}_{i j c} \boldsymbol{P}_{i c} \boldsymbol{q}_{i j c}\right\|\right)=\infty$ with $\boldsymbol{P}_{i c}=\left.\boldsymbol{P}_{i}\right|_{\boldsymbol{\eta}_{i}=\boldsymbol{\eta}_{i c}}$ and $\lambda_{\max }\left(\boldsymbol{C}_{1}\right)$ the maximum eigenvalue of $\boldsymbol{C}_{1}$. This means that we can choose a control gain matrix $\boldsymbol{C}_{1}$ such that the matrix $\boldsymbol{C}_{1}+N^{*} \varphi_{i j c}^{\prime} \boldsymbol{\Phi}_{i j c}$ is negative definite for some $(i, j)$ with $i \neq j$.

Let $\mathbb{N}^{* *} \subset \mathbb{N}^{*}$ be a nonempty set such that for all $(i, j) \in$ $\mathbb{N}^{* *}, i \neq j$, the matrix $\boldsymbol{C}_{1}+N^{*} \varphi_{i j c}^{\prime} \boldsymbol{\Phi}_{i j c}$ is negative definite. Since $\mathbb{N}^{* *} \subset \mathbb{N}^{*}$, we have $\dot{\boldsymbol{q}}_{i d}^{*}=0$, for all $i \in \mathbb{N}^{* *}$.

To investigate stability of (68) at $\boldsymbol{\xi}_{c}$, we consider the following function for the agents belonging to the set $\mathbb{N}^{* *}$ :

$$
\begin{aligned}
\bar{V}_{c}^{* *}= & \frac{1}{2} \sum_{(i, j) \in \mathbb{N}^{* *}}\left(\boldsymbol{q}_{i j}-\boldsymbol{q}_{i j c}\right)^{T} \boldsymbol{K}_{1}^{-1}\left(\boldsymbol{q}_{i j}-\boldsymbol{q}_{i j c}\right)+ \\
& \frac{1}{2} \sum_{i=1}^{N^{* *}}\left\|\boldsymbol{\eta}_{i}-\boldsymbol{\eta}_{i c}\right\|^{2}
\end{aligned}
$$

whose derivative along the solutions of (68) is

$$
\dot{\bar{V}}_{c}^{* *}=-\sum_{(i, j) \in \mathbb{N}^{* *}} W_{i j c}-\sum_{i=1}^{N^{* *}} U_{1 i}-N^{* *} \sum_{(i, j) \in \mathbb{N}^{* *}} U_{2 i j},
$$

where

$$
\begin{aligned}
& W_{i j c}=\left(\boldsymbol{q}_{i j}-\boldsymbol{q}_{i j c}\right)^{T}\left(\boldsymbol{C}_{1}+N^{* *} \varphi_{i j c}^{\prime} \boldsymbol{\Phi}_{i j c}\right)\left(\boldsymbol{q}_{i j}-\boldsymbol{q}_{i j c}\right) \\
& U_{1 i}=\left(\boldsymbol{\eta}_{i}-\boldsymbol{\eta}_{i c}\right)^{T} \boldsymbol{K}_{2}\left(\boldsymbol{\Xi}_{i}-\boldsymbol{\Xi}_{i c}\right) \\
& U_{2 i j}=\left(\boldsymbol{q}_{i j}-\boldsymbol{q}_{i j c}\right)^{T}\left(\varphi_{i j}^{\prime} \boldsymbol{\Phi}_{i j}-\varphi_{i j c}^{\prime} \boldsymbol{\Phi}_{i j c}\right) \boldsymbol{q}_{i j}
\end{aligned}
$$

We now define a set $\Psi$ such that

$$
\Psi=\left\{\left(\boldsymbol{q}_{i j}, \boldsymbol{\eta}_{i}\right) \in B_{r} \mid U_{1} \leq 0 U_{2} \leq 0, \forall(i, j) \in \mathbb{N}^{* *}, i \neq j,\right.
$$

where $U_{1}=\sum_{i=1}^{N^{* *}} U_{1 i}$ and $U_{2}=\sum_{(i, j) \in \mathbb{N}^{* *}} U_{2 i j}$. Since the matrix $\boldsymbol{C}_{1}+N^{*} \varphi_{i j c}^{\prime} \boldsymbol{\Phi}_{i j c}$ is negative definite, there exists a positive constant $\rho_{i j}$ such that

$$
\dot{\bar{V}}_{c}^{* *} \geq-\sum_{(i, j) \in \mathbb{N}^{* *}} \rho_{i j} W_{i j c},
$$

in the set $\Psi$ defined in (73). We need to show that the set $\Psi$ is nonempty.

For the condition $U_{1 i} \leq 0$, we can always find $\boldsymbol{\eta}_{i}$ as a vector function of $\boldsymbol{q}_{i j c}, \boldsymbol{q}_{i j}, \boldsymbol{\eta}_{i j c}, \boldsymbol{\eta}_{i j}$, and $\boldsymbol{\eta}_{i c}$ for all $(i, j) \in \mathbb{N}^{* *}$ and $i \neq j$ such that $U_{1 i} \leq 0$. An example is $\boldsymbol{\eta}_{i}=\boldsymbol{\eta}_{i c}$ for all $i \in \mathbb{N}^{* *}$.

For the condition $U_{2 i j} \leq 0$, we first note that the matrix $\boldsymbol{\Phi}_{i j}$ is positive definite for all $\boldsymbol{q}_{i j} \in$ $\mathbb{R}^{3}, \boldsymbol{\eta}_{i j} \in \mathbb{R}^{3}$, and $\boldsymbol{\eta}_{i} \in \mathbb{R}^{3}$ such that $\Delta_{i j}>0$, for all $(i, j) \in \mathbb{N}^{* *}$ and $i \neq$ $j$. Second, we note from Property 2) of the function $\varphi_{i j}$ in (19) that $\varphi_{i j}^{\prime}$ is negative and equals infinity when $\Delta_{i j}=0$.

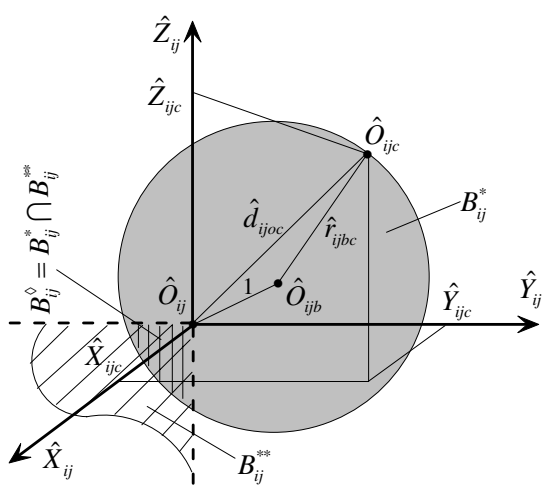

Fig. 8. An unstable set.
Now, let us choose a contact point $\hat{O}_{i j b}$, see Fig. 8, between the agent $i$ and the agent $j$ belonging to the set $\mathbb{N}^{* *}$, i.e., the point where $\Delta_{i j}=0$ such that the distance from $\hat{O}_{i j b}$ to the point $\hat{O}_{i j c}$ at $\left(\hat{X}_{i j c}, \hat{Y}_{i j c}, \hat{Z}_{i j c}\right)$ where $\left(\hat{X}_{i j c}, \hat{Y}_{i j c}, \hat{Z}_{i j c}\right):=$ $\boldsymbol{Q}_{i j c} \boldsymbol{q}_{i j c}$ is smallest. In Fig. 8, the $\hat{O}_{i j} \hat{X}_{i j}, \hat{O}_{i j} \hat{Y}_{i j}, \hat{O}_{i j} \hat{Z}_{i j}$ axes represents the first, second, and third elements of $\boldsymbol{Q}_{i j} \boldsymbol{q}_{i j}$, respectively.

Let the ball $B_{i j}^{*}$ be centered at $\hat{O}_{i j b}$ and have the radius $\hat{r}_{i j b c}$ of the distance from the center $\hat{O}_{i j b}$ to the point $\hat{O}_{i j c}$. By construction, if $\left(\boldsymbol{q}_{i j}, \boldsymbol{\eta}_{i}\right) \in B_{i j}^{*}$ with $\boldsymbol{\eta}_{i}$ being satisfied the condition $U_{1 i} \leq 0$, the matrix $\boldsymbol{S}_{i j}:=\left(\boldsymbol{Q}_{i j}^{-T}\left(\varphi_{i j}^{\prime} \boldsymbol{\Phi}_{i j}-\right.\right.$ $\left.\left.\varphi_{i j c}^{\prime} \boldsymbol{\Phi}_{i j c}\right) \boldsymbol{Q}_{i j}^{-1}\right)$ is negative definite.

On the other hand, let $B_{i j}^{* *}$ be the set such that if $\left(\boldsymbol{q}_{i j}, \boldsymbol{\eta}_{i}\right) \in$ $B_{i j}^{* *}$ then $\left[\boldsymbol{Q}_{i j}\left(\boldsymbol{P}_{i} \boldsymbol{q}_{i j}-\boldsymbol{P}_{i c} \boldsymbol{q}_{i j c}\right)\right]^{T} \boldsymbol{Q}_{i j} \boldsymbol{P}_{i} \boldsymbol{q}_{i j}$ is positive, see Fig. 8 for an illustration. Let $B_{i j}^{\diamond}=B_{i j}^{*} \cap B_{i j}^{* *}$. We can see that $B_{i j}^{\diamond}$ is nonempty if the radius $\hat{r}_{i j b c}$ is greater than 1 because the distance between the point $\hat{O}_{i j}$ and the point $\hat{O}_{i j b}$ equals 1. In order to have the radius $\hat{r}_{i j b c}>1$, we need the distance $\hat{d}_{i j o c}$ from the point $\hat{O}_{i j}$ and the point $\hat{O}_{i j c}$ larger than 2 . 
We now show that $\hat{d}_{i j o c}>2$ by choosing $\boldsymbol{C}_{1}$ with $\lambda_{\max }\left(\boldsymbol{C}_{1}\right)$ sufficiently small. As such, from (64) we can again see that $\lim _{\lambda_{\max }\left(\boldsymbol{C}_{1}\right) \rightarrow 0}\left(\left\|\boldsymbol{Q}_{i j c} \boldsymbol{P}_{i c} \boldsymbol{q}_{i j c}\right\|\right)=\infty$. This means that the set $B_{i j}^{\diamond}$ is nonempty for an appropriate $C_{1}$. Since the matrix $\boldsymbol{S}_{i j}$ is negative definite for $\left(\boldsymbol{q}_{i j}, \boldsymbol{\eta}_{i}\right) \in B_{i j}^{*}$ with $\boldsymbol{\eta}_{i}$ being satisfied the condition $U_{1 i} \leq 0$, there exists a nonempty subset $B_{i j}^{\diamond \diamond}$ of $B_{i j}^{\diamond}$ such that if $\left(\boldsymbol{q}_{i j}, \boldsymbol{\eta}_{i}\right) \in B_{i j}^{\diamond \diamond}$ with $\phi_{i}$ being satisfied the condition $U_{1 i} \leq 0$ the condition $U_{2 i j} \leq 0$ holds. Hence, the set $\Psi$ is nonempty and given by $\Psi=\bigcap_{(i, j) \in \mathbb{N}^{* *}} B_{i j}^{\diamond \diamond}$. Since we have already proved that the matrix $\boldsymbol{C}_{1}+N^{*} \varphi_{i j c}^{\prime} \boldsymbol{\Phi}_{i j c}$ is negative definite, the function $\bar{V}_{c}^{* *}$ in (71), its derivative $\dot{\bar{V}}_{c}^{* *}$ in (74) together with the nonempty set $\Psi$ imply that the undesired equilibrium set $\boldsymbol{E}_{c}$ is unstable by Chetaev's Theorem (Theorem 4.3 in [29]).

\section{REFERENCES}

[1] P. Wang, "Navigation strategies for multiple autonomous mobile robots moving in formation," Journal of Robotic Systems, vol. 8, no. 2, pp. 177 195, 1991.

[2] A. Das, R. Fierro, V. Kumar, J. Ostrowski, J. Spletzer, and C. Taylor, "A vision based formation control framework," IEEE Transactions on Robotics and Automation, vol. 18, no. 5, pp. 813-825, 2002.

[3] D. Gu and Z. Wang, "Leaderfollower flocking: Algorithms and experiments," IEEE Transactions on Control Systems Technology, vol. 17, no. 5, pp. 1211-1219, 2009.

[4] J. Hu and G. Feng, "Distributed tracking control of leaderfollower multiagent systems under noisy measurement," Automatica, vol. 46, no. 8 , pp. 1382-1387, 2010.

[5] M. Egerstedt and X. Hu, "Formation constrained multiagent control," IEEE Transactions on Robotics and Austomation, vol. 17, no. 6, pp. 947951, 2001

[6] T. Balch and R. C. Arkin, "Behavior-based formation control for multirobot teams," IEEE Transactions on Robotics and Automation, vol. 14, no. 6, pp. 926-939, 1998.

[7] R. T. Jonathan, R. W. Beard, and B. Young, "A decentralized approach to formation maneuvers," IEEE Transactions on Robotics and Automation, vol. 19, no. 6, pp. 933-941, 2003.

[8] P. Ogren, E. Fiorelli, and N. E. Leonard, "Cooperative control of mobile sensor networks: Adaptive gradient climbing in a distributed environment," IEEE Transactions on Automatic Control, vol. 49, no. 8, pp. 1292-1302, 2004.

[9] H. G. Tanner and A. Kumar, "Towards decentralization of multi-robot navigation functions," in Proceedings of the 2005 IEEE International Conference on Robotics and Automation, (Barcelona, Spain), pp. 41324137, 2005.

[10] K. D. Do, "Bounded controllers for formation stabilization of mobile agents with limited sensing ranges," IEEE Transactions on Automatic Control, vol. 52, no. 3, pp. 569-576, 2007.

[11] E. Rimon and D. E. Koditschek, "Exact robot navigation using artificial potential functions," IEEE Trans. Robot. and Automat., vol. 8, no. 5, pp. 501-518, 1992.

[12] D. M. Stipanovic, G. Inalhan, R. Teo, and C. J. Tomlin, "Decentralized overlapping control of a formation of unmanned aerial vehicles," Automatica, vol. 40, no. 8, pp. 1285-1296, 2004.

[13] R. Olfati-Saber, "Flocking for multi-agent dynamic systems: algorithms and theory," IEEE Transactions on Automatic Control, vol. 51, no. 3, pp. 401-420, 2006.

[14] D. V. Dimarogonas, S. G. Loizou, K. J. Kyriakopoulos, and M. M. Zavlanos, "A feedback stabilization and collision avoidance scheme for multiple independent non-point agents," Automatica, vol. 42, no. 2, pp. 229-243, 2006.

[15] I. Hussein and D. Stipanovic, "Effective coverage control for mobile sensor networks with guaranteed collision avoidance," IEEE Transactions on Control Systems Technology, vol. 15, no. 4, pp. 642-657, 2007.

[16] I. Hussein and A. Bloch, "Optimal control of underactuated nonholonomic mechanical systems," IEEE Transactions on Automatic Control, vol. 53, no. 3, pp. 668-681, 2008.

[17] F. Cucker and J. G. Dong, "Avoiding collisions in flocks," IEEE Transactions on Automatic Control, vol. 55, no. 5, pp. 1238-1243, 2010.
[18] A. Abdessameuda and A. Tayebi, "On consensus algorithms for doubleintegrator dynamics without velocity measurements and with input constraints," Systems and Control Letters, vol. 59, no. 5, pp. 812-821, 2010.

[19] X. Zheng, W. Iglesias, and P. Palffy-Muhoray, "Distance of closest approach of two arbitrary hard ellipsoids," Physical Review, vol. 79, p. $057702,2009$.

[20] W. Wang, Y. Choi, B. Chan, M. Kim, and J. Wang, "Efficient collision detection for moving ellipsoids using separating planes," Computing, vol. 72, pp. 235-246, 2004.

[21] X. Zheng and P. Palffy-Muhoray, "Distance of closest approach of two arbitrary hard ellipses in two dimensions," Physical Review E, vol. 75, pp. 0617091-0617096, 2007.

[22] Y. Choi, J. Chang, W. Wang, M. Kim, and G. Elber, "Continuous collision detection for ellipsoids," IEEE Transactions on Visualization and Computer Graphics, vol. 15, no. 2, pp. 311-325, 2004.

[23] B. Anderson, C. Yu, B. Fidan, and J. Hendrickx, "Rigid graph control architectures for autonomous formations," IEEE Control Systems Magazine, vol. 28, no. 6, pp. 48-63, 2008.

[24] L. Krick, M. Broucke, and B. Francis, "Stabilization of infinitesimally rigid formations of multi-robot networks," International Journal of Control, vol. 82, no. 3, pp. 423-439, 2009.

[25] M. Basiri, A. Bishop, and P. Jensfelt, "Distributed control of triangular formations with angle-only constraints," Systems and Control Letters, vol. 59, no. 2, pp. 147-154, 2010.

[26] D. Dimarogonas and K. Johansson, "Stability analysis for multi-agent systems using the incidence matrix: quantized communication and formation control," Automatica, vol. 46, no. 4, pp. 695-700, 2010.

[27] M. Cao, C. Yu, and B. Anderson, "Formation control using range-only measurements," Automatica, vol. 47, no. 4, pp. 776-781, 2011.

[28] K. D. Do, "Output-feedback formation tracking control of unicycle-type mobile robots with limited sensing ranges," Robotics and Autonomous Systems, vol. 57, pp. 34-47, 2009.

[29] H. Khalil, Nonlinear Systems. Prentice Hall, 2002.

[30] M. Krstic, I. Kanellakopoulos, and P. Kokotovic, Nonlinear and Adaptive Control Design. New York: Wiley, 1995.

[31] D. Liberzon, Switching in Systems and Control. Birkauser, 2003.

[32] K. D. Do and J. Pan, Control of Ships and Underwater Vehicles: Design for Underactuated and Nonlinear Marine Systems. Springer, 2009.

[33] C. T. Kelley, Solving Nonlinear Equations with Newton's Method. SIAM, 2003. 